\title{
Analysis and application of the nodal discontinuous Galerkin method for wave propagation in metamaterials
}

\author{
Jichun $\mathrm{Li}^{1}{ }^{*}$ and Jan Hesthaven ${ }^{2 \dagger}$ \\ ${ }^{1}$ Department of Mathematical Sciences, University of Nevada Las Vegas, \\ Las Vegas, Nevada 89154-4020, USA. \\ 2 Division of Applied Mathematics, \\ Brown University, Providence, RI 02912, USA.
}

November 26, 2012

\begin{abstract}
In this paper, we develop a nodal discontinuous Galerkin method for solving the timedependent Maxwell's equations when metamaterials are involved. Both semi- and fully-discrete schemes are constructed. Numerical stability and error estimate are proved for both schemes. Numerical results are presented to demonstrate that the method is not only efficient but also very effective in solving metamaterial Maxwell's equations.
\end{abstract}

Mathematics Subject Classification (2000): 78M10, 65N30, 35L15.

Keywords - Maxwell's equations, metamaterial, discontinuous Galerkin method, perfectly matched layers.

\section{Introduction}

Since early 2000s, the discontinuous Galerkin (DG) method has become one of the most popular methods [1] in solving various differential equations due to its flexibility in mesh construction and its convenience in parallel implementation. In the mean time, there have been considerable interests in developing DG methods for Maxwell's equations in free space $[2,5,6,7,9,15]$. Very recently, there are some DG work $[14,16,19]$ carried out for Maxwell's equations in dispersive media, whose permittivity depends on the wave frequency. However, the study of DG method for Maxwell's equations in metamaterials is quite limited, except our early work [11].

The metamaterials are artificially structured electromagnetic composite materials with some exotic properties such as negative refractive index and amplification of evanescent waves. Intensive study of metamaterials started around 2000, immediately after the successful construction of such a metamaterial by Smith et al [17]. Many potential revolutionary applications (such as construction of a perfect lens, sub-wavelength imaging and cloaking devices) have attracted researchers from many areas to work in metamaterials. Numerical simulation plays a very important role in the study of metamaterials. However, such simulations are almost exclusively based on either the classic finite-difference time-domain (FDTD) method or commercial packages such as COMSOL,

*Email: jichun@unlv.nevada.edu, Phone: (702)895-0365. Supported by National Science Foundation grant DMS0810896 .

†Email: Jan.Hesthaven@Brown.edu 
a multiphysics finite element package. Due to the constraint of FDTD method (e.g., difficult in handling the complex geometries) and the black-box characteristics of commercial packages, there is an urgent call for developing more efficient and reliable software for metamaterial simulations. In recent years, we made some initial effort $[11,12,13]$ in developing and analyzing some finite element methods (FEM) for solving the time-domain Maxwell's equations in metamaterials.

This paper continuous our initial effort [11] on developing time-domain DG methods for solving the Maxwell's equations in metamaterials. In [11], we extended the nodal DG method of Hesthaven and Warburton $[7,8]$ to metamaterial Maxwell's equations and performed some numerical tests. A major contribution of this paper is that we carry out detailed theoretical analysis of the nodal DG method applied to metamaterial Maxwell's equations. Practical numerical simulation of backward wave propagation phenomenon is also demonstrated using the newly developed nodal DG method.

The rest of the paper is organized as follows. In Sect. 2, we first present the governing equations for metamaterials, and then develop both semi- and full-discrete nodal DG methods for the metamaterial Maxwell's equations. Stability and error analysis are provided. In Sect. 3, we extend the algorithm and analysis to a perfectly matched layer (PML) model, which is used for the simulation of backward wave propagation in metamaterials. In Sect. 4, numerical results are presented to support our theoretical analysis and demonstrate the efficiency and effectiveness of the nodal DG method for modeling wave propagation in metamaterials. Sect. 5 concludes the paper..

\section{The DG method for metamaterials}

\subsection{The governing equations}

The governing equations for modeling wave propagation in metamaterials are [11]:

$$
\begin{aligned}
& \epsilon_{0} \frac{\partial \boldsymbol{E}}{\partial t}=\nabla \times \boldsymbol{H}-\boldsymbol{J}, \quad \text { in } \Omega \times(0, T), \\
& \mu_{0} \frac{\partial \boldsymbol{H}}{\partial t}=-\nabla \times \boldsymbol{E}-\boldsymbol{K}, \quad \text { in } \Omega \times(0, T), \\
& \frac{\partial \boldsymbol{J}}{\partial t}+\Gamma_{e} \boldsymbol{J}=\epsilon_{0} \omega_{p e}^{2} \boldsymbol{E}, \quad \text { in } \Omega \times(0, T), \\
& \frac{\partial \boldsymbol{K}}{\partial t}+\Gamma_{m} \boldsymbol{K}=\mu_{0} \omega_{p m}^{2} \boldsymbol{H}, \quad \text { in } \Omega \times(0, T),
\end{aligned}
$$

where $\epsilon_{0}$ denotes the permittivity of free space and $\mu_{0}$ denotes the permeability of free space, $\omega_{p e}$ and $\omega_{p m}$ are the electric and magnetic plasma frequencies respectively, $\Gamma_{e}$ and $\Gamma_{m}$ are the electric and magnetic damping frequencies respectively, $\boldsymbol{E}(\boldsymbol{x}, t)$ and $\boldsymbol{H}(\boldsymbol{x}, t)$ are the electric and magnetic fields respectively, and $\boldsymbol{J}(\boldsymbol{x}, t)$ and $\boldsymbol{K}(\boldsymbol{x}, t)$ are the induced electric and magnetic currents respectively. To make the problem well-posed, we simply assume that the boundary of $\Omega$ is perfect conducting:

$$
\hat{\boldsymbol{n}} \times \boldsymbol{E}=0 \quad \text { on } \partial \Omega,
$$

where $\hat{\boldsymbol{n}}$ is the unit outward normal to $\partial \Omega$. Furthermore, we assume that the initial conditions are

$$
\begin{array}{ll}
\boldsymbol{E}(\mathbf{x}, 0)=\boldsymbol{E}_{0}(\mathbf{x}), & \boldsymbol{H}(\mathbf{x}, 0)=\boldsymbol{H}_{0}(\mathbf{x}), \\
\boldsymbol{J}(\mathbf{x}, 0)=\boldsymbol{J}_{0}(\mathbf{x}), & \boldsymbol{K}(\mathbf{x}, 0)=\boldsymbol{K}_{0}(\mathbf{x}),
\end{array}
$$

where $\boldsymbol{E}_{0}, \boldsymbol{H}_{0}, \boldsymbol{J}_{0}$ and $\boldsymbol{K}_{0}$ are some given functions. 


\subsection{The semi-discrete DG method}

To discretize the system (1)-(4), we consider a shape-regular mesh $T_{h}$ that partitions the domain $\Omega$ into disjoint triangular elements $\left\{T_{i}\right\}$, such that $\bar{\Omega}=\bigcup_{i=1}^{N_{T}} T_{i}$. Furthermore, we denote $a_{i k}=T_{i} \cap T_{k}$ for an interior face between two elements $T_{i}$ and $T_{k}$, and $\boldsymbol{n}_{i k}$ for the unit normal vector pointed from $T_{i}$ to $T_{k}$. For any given element $T_{i}$, we denote $\nu_{i}$ for the set of all neighboring elements of $T_{i}$.

In DG methods, we consider the discretization space given by discontinuous piecewise polynomials of degree $k$ on each element, i.e.,

$$
\boldsymbol{V}_{h}=\left\{\boldsymbol{v}_{h} \in\left(L^{2}(\Omega)\right)^{d}:\left.\quad \boldsymbol{v}_{h}\right|_{T_{i}} \in\left(P_{k}\right)^{d}, \quad \forall T_{i} \in T_{h}\right\}, \quad d=2 \text { or } 3 .
$$

Multiplying equations (1)-(4) by test functions $\boldsymbol{u}, \boldsymbol{v}, \boldsymbol{\phi}, \boldsymbol{\psi}$, respectively, and integrating by parts over any element $T_{i} \in T_{h}$, we have

$$
\begin{aligned}
& \epsilon_{0} \int_{T_{i}} \frac{\partial \boldsymbol{E}}{\partial t} \cdot \boldsymbol{u}-\int_{T_{i}} \boldsymbol{H} \cdot \nabla \times \boldsymbol{u}-\int_{\partial T_{i}} \boldsymbol{n}_{i} \times \boldsymbol{H} \cdot \boldsymbol{u}+\int_{T_{i}} \boldsymbol{J} \cdot \boldsymbol{u}=0, \\
& \mu_{0} \int_{T_{i}} \frac{\partial \boldsymbol{H}}{\partial t} \cdot \boldsymbol{v}+\int_{T_{i}} \boldsymbol{E} \cdot \nabla \times \boldsymbol{v}+\int_{\partial T_{i}} \boldsymbol{n}_{i} \times \boldsymbol{E} \cdot \boldsymbol{v}+\int_{T_{i}} \boldsymbol{K} \cdot \boldsymbol{v}=0, \\
& \frac{1}{\epsilon_{0} \omega_{p e}^{2}} \int_{T_{i}} \frac{\partial \boldsymbol{J}}{\partial t} \cdot \boldsymbol{\phi}+\frac{\Gamma_{e}}{\epsilon_{0} \omega_{p e}^{2}} \int_{T_{i}} \boldsymbol{J} \cdot \boldsymbol{\phi}=\int_{T_{i}} \boldsymbol{E} \cdot \boldsymbol{\phi} \\
& \frac{1}{\mu_{0} \omega_{p m}^{2}} \int_{T_{i}} \frac{\partial \boldsymbol{K}}{\partial t} \cdot \boldsymbol{\psi}+\frac{\Gamma_{m}}{\mu_{0} \omega_{p m}^{2}} \int_{T_{i}} \boldsymbol{K} \cdot \boldsymbol{\psi}=\int_{T_{i}} \boldsymbol{H} \cdot \boldsymbol{\psi} .
\end{aligned}
$$

Let us look at the semi-discrete solution $\boldsymbol{E}_{h}, \boldsymbol{H}_{h}, \boldsymbol{J}_{h}, \boldsymbol{K}_{h} \in C^{1}\left(0, T ; \boldsymbol{V}_{h}\right)$ as a solution of the following weak formulation: For any $\boldsymbol{u}_{h}, \boldsymbol{v}_{h}, \boldsymbol{\phi}_{h}, \boldsymbol{\psi}_{h} \in \boldsymbol{V}_{h}$, and any element $T_{i} \in T_{h}$,

$$
\begin{aligned}
& \epsilon_{0} \int_{T_{i}} \frac{\partial \boldsymbol{E}_{h}}{\partial t} \cdot \boldsymbol{u}_{h}-\int_{T_{i}} \boldsymbol{H}_{h} \cdot \nabla \times \boldsymbol{u}_{h}-\sum_{K \in \nu_{i}} \int_{a_{i k}} \boldsymbol{u}_{h} \cdot \boldsymbol{n}_{i k} \times\left\{\left\{\boldsymbol{H}_{h}\right\}\right\}_{i k}+\int_{T_{i}} \boldsymbol{J}_{h} \cdot \boldsymbol{u}_{h}=0, \\
& \mu_{0} \int_{T_{i}} \frac{\partial \boldsymbol{H}_{h}}{\partial t} \cdot \boldsymbol{v}_{h}+\int_{T_{i}} \boldsymbol{E}_{h} \cdot \nabla \times \boldsymbol{v}_{h}+\sum_{K \in \nu_{i}} \int_{a_{i k}} \boldsymbol{v}_{h} \cdot \boldsymbol{n}_{i k} \times\left\{\left\{\boldsymbol{E}_{h}\right\}\right\}_{i k}+\int_{T_{i}} \boldsymbol{K}_{h} \cdot \boldsymbol{v}_{h}=0, \\
& \frac{1}{\epsilon_{0} \omega_{p e}^{2}} \int_{T_{i}} \frac{\partial \boldsymbol{J}_{h}}{\partial t} \cdot \boldsymbol{\phi}_{h}+\frac{\Gamma_{e}}{\epsilon_{0} \omega_{p e}^{2}} \int_{T_{i}} \boldsymbol{J}_{h} \cdot \boldsymbol{\phi}_{h}=\int_{T_{i}} \boldsymbol{E}_{h} \cdot \boldsymbol{\phi}_{h}, \\
& \frac{1}{\mu_{0} \omega_{p m}^{2}} \int_{T_{i}} \frac{\partial \boldsymbol{K}_{h}}{\partial t} \cdot \boldsymbol{\psi}_{h}+\frac{\Gamma_{m}}{\mu_{0} \omega_{p m}^{2}} \int_{T_{i}} \boldsymbol{K}_{h} \cdot \boldsymbol{\psi}_{h}=\int_{T_{i}} \boldsymbol{H}_{h} \cdot \boldsymbol{\psi}_{h},
\end{aligned}
$$

hold true and are subject to the initial conditions:

$$
\boldsymbol{E}_{h}(0)=\Pi_{2} \boldsymbol{E}_{0}(\boldsymbol{x}), \boldsymbol{H}_{h}(0)=\Pi_{2} \boldsymbol{H}_{0}(\boldsymbol{x}), \boldsymbol{J}_{h}(0)=\Pi_{2} \boldsymbol{J}_{0}(\boldsymbol{x}), \boldsymbol{K}_{h}(0)=\Pi_{2} \boldsymbol{K}_{0}(\boldsymbol{x}),
$$

where $\Pi_{2}$ denotes the standard $L^{2}$-projection onto $\boldsymbol{V}_{h}$. Here and below for any function $\boldsymbol{v}_{h}$ we denote the average and jump through any internal face $a_{i k}$ as

$$
\left\{\left\{\boldsymbol{v}_{h}\right\}\right\}_{i k}=\frac{1}{2}\left(\boldsymbol{v}_{i}+\boldsymbol{v}_{k}\right), \quad\left[\left[\boldsymbol{v}_{h}\right]\right]_{i k}=\left(\boldsymbol{v}_{k}-\boldsymbol{v}_{i}\right)
$$

Note that the perfect conducting boundary condition (5) is treated as $\left.\boldsymbol{E}_{k}\right|_{a_{i k}}=-\left.\boldsymbol{E}_{i}\right|_{a_{i k}}$ and $\left.\boldsymbol{H}_{k}\right|_{a_{i k}}=\left.\boldsymbol{H}_{i}\right|_{a_{i k}}$, which lead to

$$
\{\{\boldsymbol{E}\}\}_{i k}=0, \quad \text { and } \quad\{\{\boldsymbol{H}\}\}_{i k}=\left.\boldsymbol{H}_{i}\right|_{a_{i k}} \quad \text { for any } a_{i k} \in \partial \Omega .
$$


Here and below we denote $\boldsymbol{E}_{i}=\left.\boldsymbol{E}_{h}\right|_{T_{i}}, \boldsymbol{H}_{i}=\left.\boldsymbol{H}_{h}\right|_{T_{i}}, \boldsymbol{J}_{i}=\left.\boldsymbol{J}_{h}\right|_{T_{i}}$ and $\boldsymbol{K}_{i}=\left.\boldsymbol{K}_{h}\right|_{T_{i}}$.

Denote the semi-discrete energy $\mathcal{E}_{h}$ :

$$
\mathcal{E}_{h}(t)=\frac{1}{2}\left(\epsilon_{0}\left\|\boldsymbol{E}_{h}(t)\right\|_{0, \Omega}^{2}+\mu_{0}\left\|\boldsymbol{H}_{h}(t)\right\|_{0, \Omega}^{2}+\frac{1}{\epsilon_{0} \omega_{p e}^{2}}\left\|\boldsymbol{J}_{h}(t)\right\|_{0, \Omega}^{2}+\frac{1}{\mu_{0} \omega_{p m}^{2}}\left\|\boldsymbol{K}_{h}(t)\right\|_{0, \Omega}^{2}\right),
$$

and a bilinear form $\mathcal{B}_{i}$ :

$$
\begin{aligned}
\mathcal{B}_{i}(\boldsymbol{E}, \boldsymbol{H})= & -\int_{T_{i}} \boldsymbol{H}_{i} \cdot \nabla \times \boldsymbol{E}_{i}-\sum_{K \in \nu_{i}} \int_{a_{i k}} \boldsymbol{E}_{h} \cdot \boldsymbol{n}_{i k} \times\left\{\left\{\boldsymbol{H}_{h}\right\}\right\}_{i k} \\
& +\int_{T_{i}} \boldsymbol{E}_{i} \cdot \nabla \times \boldsymbol{H}_{i}+\sum_{K \in \nu_{i}} \int_{a_{i k}} \boldsymbol{H}_{h} \cdot \boldsymbol{n}_{i k} \times\left\{\left\{\boldsymbol{E}_{h}\right\}\right\}_{i k} .
\end{aligned}
$$

Theorem 2.1 The energy $\mathcal{E}_{h}$ is decreasing in time, i.e., $\mathcal{E}_{h}(t) \leq \mathcal{E}_{h}(0)$.

Proof. Choosing $\boldsymbol{u}_{h}=\boldsymbol{E}_{h}, \boldsymbol{v}_{h}=\boldsymbol{H}_{h}, \phi_{h}=\boldsymbol{J}_{h}, \boldsymbol{\psi}_{h}=\boldsymbol{K}_{h}$ in (12)-(15), respectively, and adding the results over all element $T_{i} \in T_{h}$, we obtain

$$
\frac{d}{d t} \mathcal{E}_{h}(t)+\frac{\Gamma_{e}}{\epsilon_{0} \omega_{p e}^{2}}\left\|\boldsymbol{J}_{h}(t)\right\|_{0, \Omega}^{2}+\frac{\Gamma_{m}}{\mu_{0} \omega_{p m}^{2}}\left\|\boldsymbol{K}_{h}(t)\right\|_{0, \Omega}^{2}+\sum_{i} \mathcal{B}_{i}(\boldsymbol{E}, \boldsymbol{H})=0 .
$$

By the definition of $\mathcal{B}_{i}$ and integration by parts, we have

$$
\begin{aligned}
\mathcal{B}_{i}(\boldsymbol{E}, \boldsymbol{H})= & \sum_{K \in \nu_{i}} \int_{a_{i k}} \boldsymbol{E}_{i} \cdot \boldsymbol{n}_{i k} \times \boldsymbol{H}_{i} \\
& \quad+\sum_{K \in \nu_{i}} \int_{a_{i k}} \boldsymbol{E}_{i} \cdot\left\{\left\{\boldsymbol{H}_{h}\right\}\right\}_{i k} \times \boldsymbol{n}_{i k}-\sum_{K \in \nu_{i}} \int_{a_{i k}} \boldsymbol{H}_{i} \cdot\left\{\left\{\boldsymbol{E}_{h}\right\}\right\}_{i k} \times \boldsymbol{n}_{i k} \\
= & \sum_{K \in \nu_{i}} \int_{a_{i k}}\left[-\boldsymbol{E}_{i} \times \boldsymbol{H}_{i}+\boldsymbol{E}_{i} \times \frac{\boldsymbol{H}_{i}+\boldsymbol{H}_{k}}{2}-\boldsymbol{H}_{i} \times \frac{\boldsymbol{E}_{i}+\boldsymbol{E}_{k}}{2}\right] \cdot \boldsymbol{n}_{i k} \\
= & \frac{1}{2} \sum_{K \in \nu_{i}} \int_{a_{i k}}\left(\boldsymbol{E}_{i} \times \boldsymbol{H}_{k}+\boldsymbol{E}_{k} \times \boldsymbol{H}_{i}\right) \cdot \boldsymbol{n}_{i k} .
\end{aligned}
$$

From (20), we obtain $\sum_{i} \mathcal{B}_{i}(\boldsymbol{E}, \boldsymbol{H})=0$, which, along with (19), concludes the proof.

For the semi-discrete scheme (12)-(15), we have the following convergence result.

Theorem 2.2 If $\boldsymbol{E}, \boldsymbol{H}, \boldsymbol{J}, \boldsymbol{K} \in C^{0}\left([0, T] ;\left(H^{s+1}(\Omega)\right)^{d}\right)$ for $s \geq 0$, then there exists a constant $C>0$ independent of $h$ such that

$$
\begin{aligned}
& \max _{t \in[0, T]}\left(\left\|\boldsymbol{E}-\boldsymbol{E}_{h}\right\|_{0, \Omega}+\left\|\boldsymbol{H}-\boldsymbol{H}_{h}\right\|_{0, \Omega}+\left\|\boldsymbol{J}-\boldsymbol{J}_{h}\right\|_{0, \Omega}+\left\|\boldsymbol{K}-\boldsymbol{K}_{h}\right\|_{0, \Omega}\right) \\
\leq & C h^{\min (s, k)}\|(\boldsymbol{E}, \boldsymbol{H}, \boldsymbol{J}, \boldsymbol{K})\|_{C^{0}\left([0, T] ;\left(H^{s+1}(\Omega)\right)^{d}\right)} .
\end{aligned}
$$

Proof. Let us introduce the notation $\tilde{\boldsymbol{W}}_{h}=P_{h}(\boldsymbol{W})-\boldsymbol{W}_{h}$ and $\overline{\boldsymbol{W}}_{h}=P_{h}(\boldsymbol{W})-\boldsymbol{W}$ for $\boldsymbol{W}=\boldsymbol{E}, \boldsymbol{H}, \boldsymbol{J}, \boldsymbol{K}$. 
Subtracting (12)-(15) from (8)-(11), we have the error equations:

$$
\begin{aligned}
& \epsilon_{0} \int_{T_{i}} \frac{\partial \tilde{\boldsymbol{E}}_{h}}{\partial t} \cdot \boldsymbol{u}_{h}-\int_{T_{i}} \tilde{\boldsymbol{H}}_{h} \cdot \nabla \times \boldsymbol{u}_{h}-\sum_{K \in \nu_{i}} \int_{a_{i k}} \boldsymbol{u}_{h} \cdot \boldsymbol{n}_{i k} \times\left\{\left\{\tilde{\boldsymbol{H}}_{h}\right\}\right\}_{i k}+\int_{T_{i}} \tilde{\boldsymbol{J}}_{h} \cdot \boldsymbol{u}_{h} \\
& =\epsilon_{0} \int_{T_{i}} \frac{\partial \overline{\boldsymbol{E}}_{h}}{\partial t} \cdot \boldsymbol{u}_{h}-\int_{T_{i}} \overline{\boldsymbol{H}}_{h} \cdot \nabla \times \boldsymbol{u}_{h}-\sum_{K \in \nu_{i}} \int_{a_{i k}} \boldsymbol{u}_{h} \cdot \boldsymbol{n}_{i k} \times\left\{\left\{\overline{\boldsymbol{H}}_{h}\right\}\right\}_{i k}+\int_{T_{i}} \overline{\boldsymbol{J}}_{h} \cdot \boldsymbol{u}_{h}, \\
& \mu_{0} \int_{T_{i}} \frac{\partial \tilde{\boldsymbol{H}}_{h}}{\partial t} \cdot \boldsymbol{v}_{h}+\int_{T_{i}} \tilde{\boldsymbol{E}}_{h} \cdot \nabla \times \boldsymbol{v}_{h}+\sum_{K \in \nu_{i}} \int_{a_{i k}} \boldsymbol{v}_{h} \cdot \boldsymbol{n}_{i k} \times\left\{\left\{\tilde{\boldsymbol{E}}_{h}\right\}\right\}_{i k}+\int_{T_{i}} \tilde{\boldsymbol{K}}_{h} \cdot \boldsymbol{v}_{h} \\
& =\mu_{0} \int_{T_{i}} \frac{\partial \overline{\boldsymbol{H}}_{h}}{\partial t} \cdot \boldsymbol{v}_{h}+\int_{T_{i}} \overline{\boldsymbol{E}}_{h} \cdot \nabla \times \boldsymbol{v}_{h}+\sum_{K \in \nu_{i}} \int_{a_{i k}} \boldsymbol{v}_{h} \cdot \boldsymbol{n}_{i k} \times\left\{\left\{\overline{\boldsymbol{E}}_{h}\right\}\right\}_{i k}+\int_{T_{i}} \overline{\boldsymbol{K}}_{h} \cdot \boldsymbol{v}_{h}, \\
& \frac{1}{\epsilon_{0} \omega_{p e}^{2}} \int_{T_{i}} \frac{\partial \tilde{\boldsymbol{J}}_{h}}{\partial t} \cdot \phi_{h}+\frac{\Gamma_{e}}{\epsilon_{0} \omega_{p e}^{2}} \int_{T_{i}} \tilde{\boldsymbol{J}}_{h} \cdot \boldsymbol{\phi}_{h}-\int_{T_{i}} \tilde{\boldsymbol{E}}_{h} \cdot \boldsymbol{\phi}_{h} \\
& =\frac{1}{\epsilon_{0} \omega_{p e}^{2}} \int_{T_{i}} \frac{\partial \overline{\boldsymbol{J}}_{h}}{\partial t} \cdot \phi_{h}+\frac{\Gamma_{e}}{\epsilon_{0} \omega_{p e}^{2}} \int_{T_{i}} \overline{\boldsymbol{J}}_{h} \cdot \boldsymbol{\phi}_{h}-\int_{T_{i}} \overline{\boldsymbol{E}}_{h} \cdot \phi_{h}, \\
& \text { (iv) } \quad \frac{1}{\mu_{0} \omega_{p m}^{2}} \int_{T_{i}} \frac{\partial \tilde{\boldsymbol{K}}_{h}}{\partial t} \cdot \boldsymbol{\psi}_{h}+\frac{\Gamma_{m}}{\mu_{0} \omega_{p m}^{2}} \int_{T_{i}} \tilde{\boldsymbol{K}}_{h} \cdot \boldsymbol{\psi}_{h}-\int_{T_{i}} \tilde{\boldsymbol{H}}_{h} \cdot \boldsymbol{\psi}_{h} \\
& =\frac{1}{\mu_{0} \omega_{p m}^{2}} \int_{T_{i}} \frac{\partial \overline{\boldsymbol{K}}_{h}}{\partial t} \cdot \boldsymbol{\psi}_{h}+\frac{\Gamma_{m}}{\mu_{0} \omega_{p m}^{2}} \int_{T_{i}} \overline{\boldsymbol{K}}_{h} \cdot \boldsymbol{\psi}_{h}-\int_{T_{i}} \overline{\boldsymbol{H}}_{h} \cdot \boldsymbol{\psi}_{h} .
\end{aligned}
$$

Choosing $\boldsymbol{u}_{h}=\tilde{\boldsymbol{E}}_{h}, \boldsymbol{v}_{h}=\tilde{\boldsymbol{H}}_{h}, \phi_{h}=\tilde{\boldsymbol{J}}_{h}, \boldsymbol{\psi}_{h}=\tilde{\boldsymbol{K}}_{h}$ in (22)-(25), respectively, summing up the results for all elements $T_{i}$ of $T_{h}$, then using the projection property and the energy definition (17), we have

$$
\begin{aligned}
& \frac{d}{d t} \tilde{\mathcal{E}}_{h}+\frac{\Gamma_{e}}{\epsilon_{0} \omega_{p e}^{2}}\left\|\tilde{\boldsymbol{J}}_{h}\right\|_{0, \Omega}^{2}+\frac{\Gamma_{m}}{\mu_{0} \omega_{p m}^{2}}\left\|\tilde{\boldsymbol{K}}_{h}\right\|_{0, \Omega}^{2}+\sum_{i} \mathcal{B}_{i}(\tilde{\boldsymbol{E}}, \tilde{\boldsymbol{H}}) \\
= & \sum_{i} \sum_{K \in \nu_{i}}\left[-\int_{a_{i k}} \tilde{\boldsymbol{E}}_{h} \cdot \boldsymbol{n}_{i k} \times\left\{\left\{\overline{\boldsymbol{H}}_{h}\right\}\right\}_{i k}+\int_{a_{i k}} \tilde{\boldsymbol{H}}_{h} \cdot \boldsymbol{n}_{i k} \times\left\{\left\{\overline{\boldsymbol{E}}_{h}\right\}\right\}_{i k}\right] \\
\leq & \sum_{i}\left[\left\|\tilde{\boldsymbol{E}}_{h}\right\|_{0, \partial T_{i}}\left\|\overline{\boldsymbol{H}}_{h}\right\|_{0, \partial T_{i}}+\left\|\tilde{\boldsymbol{H}}_{h}\right\|_{0, \partial T_{i}}\left\|\overline{\boldsymbol{E}}_{h}\right\|_{0, \partial T_{i}}\right] \\
\leq & \sum_{i}\left[C h_{T_{i}}^{-\frac{1}{2}}\left\|\tilde{\boldsymbol{E}}_{h}\right\|_{0, T_{i}} C h_{T_{i}}^{\min (s, k)+\frac{1}{2}}\|\boldsymbol{H}\|_{s+1, T_{i}}+C h_{T_{i}}^{-\frac{1}{2}}\left\|\tilde{\boldsymbol{H}}_{h}\right\|_{0, T_{i}} C h_{T_{i}}^{\min (s, k)+\frac{1}{2}}\|\boldsymbol{E}\|_{s+1, T_{i}}\right],
\end{aligned}
$$

where in the last step we used the standard inverse inequality and interpolation error estimate.

The proof is completed by using the fact $\sum_{i} \mathcal{B}_{i}(\tilde{\boldsymbol{E}}, \tilde{\boldsymbol{H}})=0$ and the Gronwall inequality.

\subsection{The fully-discrete DG method}

To define a fully discrete scheme, we divide the time interval $(0, T)$ into $N$ uniform subintervals by points $0=t_{0}<t_{1}<\cdots<t_{N}=T$, where $t_{k}=k \tau$, and $\tau=T / N$.

Following the idea of our previous work on leap-frog scheme [12], we can construct a leapfrog DG scheme as follows: Given initial approximations of $\boldsymbol{E}_{h}^{0}, \boldsymbol{H}_{h}^{\frac{1}{2}}, \boldsymbol{J}_{h}^{\frac{1}{2}}, \boldsymbol{K}_{h}^{0}$, for any $n \geq 0$, find 


$$
\begin{aligned}
& \boldsymbol{E}_{h}^{n+1}, \boldsymbol{H}_{h}^{n+\frac{3}{2}}, \boldsymbol{J}_{h}^{n+\frac{3}{2}}, \boldsymbol{K}_{h}^{n+1} \in \boldsymbol{V}_{h} \text { such that } \\
& \epsilon_{0} \int_{T_{i}} \frac{\boldsymbol{E}_{h}^{n+1}-\boldsymbol{E}_{h}^{n}}{\tau} \cdot \boldsymbol{u}_{h}-\int_{T_{i}} \boldsymbol{H}_{h}^{n+\frac{1}{2}} \cdot \nabla \times \boldsymbol{u}_{h}-\sum_{K \in \nu_{i}} \int_{a_{i k}} \boldsymbol{u}_{h} \cdot \boldsymbol{n}_{i k} \times\left\{\left\{\boldsymbol{H}_{h}^{n+\frac{1}{2}}\right\}\right\}_{i k}+\int_{T_{i}} \boldsymbol{J}_{h}^{n+\frac{1}{2}} \cdot \boldsymbol{u}_{h}=0 \\
& \mu_{0} \int_{T_{i}} \frac{\boldsymbol{H}_{h}^{n+\frac{3}{2}}-\boldsymbol{H}_{h}^{n+\frac{1}{2}}}{\tau} \cdot \boldsymbol{v}_{h}+\int_{T_{i}} \boldsymbol{E}_{h}^{n+1} \cdot \nabla \times \boldsymbol{v}_{h}+\sum_{K \in \nu_{i}} \int_{a_{i k}} \boldsymbol{v}_{h} \cdot \boldsymbol{n}_{i k} \times\left\{\left\{\boldsymbol{E}_{h}^{n+1}\right\}\right\}_{i k}+\int_{T_{i}} \boldsymbol{K}_{h}^{n+1} \cdot \boldsymbol{v}_{h}=0 \\
& \frac{1}{\epsilon_{0} \omega_{p e}^{2}} \int_{T_{i}} \frac{\boldsymbol{J}_{h}^{n+\frac{3}{2}}-\boldsymbol{J}_{h}^{n+\frac{1}{2}}}{\tau} \cdot \boldsymbol{\phi}_{h}+\frac{\Gamma_{e}}{\epsilon_{0} \omega_{p e}^{2}} \int_{T_{i}} \frac{\boldsymbol{J}_{h}^{n+\frac{3}{2}}+\boldsymbol{J}_{h}^{n+\frac{1}{2}}}{2} \cdot \boldsymbol{\phi}_{h}=\int_{T_{i}} \boldsymbol{E}_{h}^{n+1} \cdot \boldsymbol{\phi}_{h}, \\
& \frac{1}{\mu_{0} \omega_{p m}^{2}} \int_{T_{i}} \frac{\boldsymbol{K}_{h}^{n+1}-\boldsymbol{K}_{h}^{n}}{\tau} \cdot \boldsymbol{\psi}_{h}+\frac{\Gamma_{m}}{\mu_{0} \omega_{p m}^{2}} \int_{T_{i}} \frac{\boldsymbol{K}_{h}^{n+1}+\boldsymbol{K}_{h}^{n}}{2} \cdot \boldsymbol{\psi}_{h}=\int_{T_{i}} \boldsymbol{H}_{h}^{n+\frac{1}{2}} \cdot \boldsymbol{\psi}_{h},
\end{aligned}
$$

hold true for any $\boldsymbol{u}_{h}, \boldsymbol{v}_{h}, \phi_{h}, \boldsymbol{\psi}_{h} \in \boldsymbol{V}_{h}$, and any element $T_{i} \in T_{h}$.

Denote $c_{v}=\frac{1}{\sqrt{\epsilon_{0} \mu_{0}}}$ for the wave propagation speed in vacuum, $c_{i n v}>0$ for the constant in the standard inverse estimate

$$
\left\|\nabla \times \phi_{h}\right\|_{0} \leq c_{i n v} h^{-1}\left\|\phi_{h}\right\|_{0} \quad \forall \phi_{h} \in V_{h},
$$

and $c_{t r}>0$ for the constant in the standard trace inequality

$$
\left\|\phi_{h}\right\|_{0, \partial \Omega} \leq c_{t r} h^{-1 / 2}\left\|\phi_{h}\right\|_{0, \Omega} \quad \forall \phi_{h} \in \boldsymbol{V}_{h} .
$$

Theorem 2.3 Under the CFL condition

$$
\tau \leq \min \left\{\frac{h}{2 c_{v} c_{i n v}}, \frac{h}{c_{t r}^{2} c_{v}}, \frac{1}{\sqrt{2} \omega_{p e}}, \frac{1}{\sqrt{2} \omega_{p m}}\right\} .
$$

the solution $\left(\boldsymbol{E}_{h}^{N+1}, \boldsymbol{H}_{h}^{N+\frac{3}{2}}, \boldsymbol{J}_{h}^{N+\frac{3}{2}}, \boldsymbol{K}_{h}^{N+1}\right)$ of (26)-(29) satisfies the following stability: For any $N \geq 0$,

$$
\begin{aligned}
& \epsilon_{0}\left\|\boldsymbol{E}_{h}^{N+1}\right\|_{0, \Omega}^{2}+\mu_{0}\left\|\boldsymbol{H}_{h}^{N+\frac{3}{2}}\right\|_{0, \Omega}^{2}+\frac{1}{\epsilon_{0} \omega_{p e}^{2}}\left\|\boldsymbol{J}_{h}^{N+\frac{3}{2}}\right\|_{0, \Omega}^{2}+\frac{1}{\mu_{0} \omega_{p m}^{2}}\left\|\boldsymbol{K}_{h}^{N+1}\right\|_{0, \Omega}^{2} \\
\leq & C\left[\epsilon_{0}\left\|\boldsymbol{E}_{h}^{0}\right\|_{0, \Omega}^{2}+\mu_{0}\left\|\boldsymbol{H}_{h}^{\frac{1}{2}}\right\|_{0, \Omega}^{2}+\frac{1}{\epsilon_{0} \omega_{p e}^{2}}\left\|\boldsymbol{J}_{h}^{\frac{1}{2}}\right\|_{0, \Omega}^{2}+\frac{1}{\mu_{0} \omega_{p m}^{2}}\left\|\boldsymbol{K}_{h}^{0}\right\|_{0, \Omega}^{2}\right],
\end{aligned}
$$

holds true, where the constant $C>0$ is independent of $h$ and $\tau$.

Proof. Choosing $\boldsymbol{u}_{h}=\frac{\tau}{2}\left(\boldsymbol{E}_{i}^{n+1}+\boldsymbol{E}_{i}^{n}\right), \boldsymbol{v}_{h}=\frac{\tau}{2}\left(\boldsymbol{H}_{i}^{n+\frac{3}{2}}+\boldsymbol{H}_{i}^{n+\frac{1}{2}}\right), \phi_{h}=\frac{\tau}{2}\left(\boldsymbol{J}_{i}^{n+\frac{3}{2}}+\boldsymbol{J}_{i}^{n+\frac{1}{2}}\right), \boldsymbol{\psi}_{h}=$ $\frac{\tau}{2}\left(\boldsymbol{K}_{i}^{n+1}+\boldsymbol{K}_{i}^{n}\right)$ in (26)-(29), respectively, we obtain

$$
\begin{aligned}
& \frac{\epsilon_{0}}{2}\left(\left\|\boldsymbol{E}_{i}^{n+1}\right\|_{0, T_{i}}^{2}-\left\|\boldsymbol{E}_{i}^{n}\right\|_{0, T_{i}}^{2}\right)+\frac{\mu_{0}}{2}\left(\left\|\boldsymbol{H}_{i}^{n+\frac{3}{2}}\right\|_{0, T_{i}}^{2}-\left\|\boldsymbol{H}_{i}^{n+\frac{1}{2}}\right\|_{0, T_{i}}^{2}\right)+\frac{1}{2 \epsilon_{0} \omega_{p e}^{2}}\left(\left\|\boldsymbol{J}_{i}^{n+\frac{3}{2}}\right\|_{0, T_{i}}^{2}-\left\|\boldsymbol{J}_{i}^{n+\frac{1}{2}}\right\|_{0, T_{i}}^{2}\right) \\
& +\frac{1}{2 \mu_{0} \omega_{p m}^{2}}\left(\left\|\boldsymbol{K}_{i}^{n+1}\right\|_{0, T_{i}}^{2}-\left\|\boldsymbol{K}_{i}^{n}\right\|_{0, T_{i}}^{2}\right)+\frac{\tau \Gamma_{e}}{\epsilon_{0} \omega_{p e}^{2}}\left\|\frac{\boldsymbol{J}_{i}^{n+\frac{3}{2}}+\boldsymbol{J}_{i}^{n+\frac{1}{2}}}{2}\right\|_{0, T_{i}}^{2}+\frac{\tau \Gamma_{m}}{\mu_{0} \omega_{p m}^{2}}\left\|\frac{\boldsymbol{K}_{i}^{n+1}+\boldsymbol{K}_{i}^{n}}{2}\right\|_{0, T_{i}}^{2} \\
= & \frac{\tau}{2}\left[\int_{T_{i}} \boldsymbol{H}_{i}^{n+\frac{1}{2}} \cdot \nabla \times\left(\boldsymbol{E}_{i}^{n+1}+\boldsymbol{E}_{i}^{n}\right)-\int_{T_{i}} \boldsymbol{E}_{i}^{n+1} \cdot \nabla \times\left(\boldsymbol{H}_{i}^{n+\frac{3}{2}}+\boldsymbol{H}_{i}^{n+\frac{1}{2}}\right)\right]
\end{aligned}
$$




$$
\begin{aligned}
& +\sum_{K \in \nu_{i}} \int_{a_{i k}} \frac{\tau}{2}\left(\boldsymbol{E}_{i}^{n+1}+\boldsymbol{E}_{i}^{n}\right) \cdot \boldsymbol{n}_{i k} \times\left\{\left\{\boldsymbol{H}_{h}^{n+\frac{1}{2}}\right\}\right\}_{i k} \\
& -\sum_{K \in \nu_{i}} \int_{a_{i k}} \frac{\tau}{2}\left(\boldsymbol{H}_{i}^{n+\frac{3}{2}}+\boldsymbol{H}_{i}^{n+\frac{1}{2}}\right) \cdot \boldsymbol{n}_{i k} \times\left\{\left\{\boldsymbol{E}_{h}^{n+1}\right\}\right\}_{i k} \\
& -\int_{T_{i}} \boldsymbol{J}_{i}^{n+\frac{1}{2}} \cdot \frac{\tau}{2}\left(\boldsymbol{E}_{i}^{n+1}+\boldsymbol{E}_{i}^{n}\right)+\int_{T_{i}} \boldsymbol{E}_{i}^{n+1} \cdot \frac{\tau}{2}\left(\boldsymbol{J}_{i}^{n+\frac{3}{2}}+\boldsymbol{J}_{i}^{n+\frac{1}{2}}\right) \\
& -\int_{T_{i}} \boldsymbol{K}_{i}^{n+1} \cdot \frac{\tau}{2}\left(\boldsymbol{H}_{i}^{n+\frac{3}{2}}+\boldsymbol{H}_{i}^{n+\frac{1}{2}}\right)+\int_{T_{i}} \boldsymbol{H}_{i}^{n+\frac{1}{2}} \cdot \frac{\tau}{2}\left(\boldsymbol{K}_{i}^{n+1}+\boldsymbol{K}_{i}^{n}\right) .
\end{aligned}
$$

Using the Stokes' formula, we have

$$
\int_{T_{i}} \boldsymbol{H}_{i}^{n+\frac{1}{2}} \cdot \nabla \times\left(\boldsymbol{E}_{i}^{n+1}+\boldsymbol{E}_{i}^{n}\right)=\int_{T_{i}} \nabla \times \boldsymbol{H}_{i}^{n+\frac{1}{2}} \cdot\left(\boldsymbol{E}_{i}^{n+1}+\boldsymbol{E}_{i}^{n}\right)-\sum_{K \in \nu_{i}} \int_{a_{i k}}\left(\boldsymbol{E}_{i}^{n+1}+\boldsymbol{E}_{i}^{n}\right) \times \boldsymbol{H}_{i}^{n+\frac{1}{2}} \cdot \boldsymbol{n}_{i k}
$$

Adding all boundary integral terms in both (34) and (35), and using the average definition, we have

$$
\begin{aligned}
\operatorname{sum}_{1 i} \equiv & \sum_{K \in \nu_{i}} \int_{a_{i k}} \frac{\tau}{2}\left(\boldsymbol{E}_{h}^{n+1}+\boldsymbol{E}_{h}^{n}\right) \cdot \boldsymbol{n}_{i k} \times\left\{\left\{\boldsymbol{H}_{h}^{n+\frac{1}{2}}\right\}\right\}_{i k} \\
& -\sum_{K \in \nu_{i}} \int_{a_{i k}} \frac{\tau}{2}\left(\boldsymbol{H}_{h}^{n+\frac{3}{2}}+\boldsymbol{H}_{h}^{n+\frac{1}{2}}\right) \cdot \boldsymbol{n}_{i k} \times\left\{\left\{\boldsymbol{E}_{h}^{n+1}\right\}\right\}_{i k} \\
& -\frac{\tau}{2} \sum_{K \in \nu_{i}} \int_{a_{i k}}\left(\boldsymbol{E}_{i}^{n+1}+\boldsymbol{E}_{i}^{n}\right) \times \boldsymbol{H}_{i}^{n+\frac{1}{2}} \cdot \boldsymbol{n}_{i k} \\
= & -\frac{\tau}{4} \sum_{K \in \nu_{i}} \int_{a_{i k}}\left[\left(\boldsymbol{E}_{i}^{n+1}+\boldsymbol{E}_{i}^{n}\right) \times\left(\boldsymbol{H}_{i}^{n+\frac{1}{2}}+\boldsymbol{H}_{k}^{n+\frac{1}{2}}\right)-\left(\boldsymbol{H}_{i}^{n+\frac{3}{2}}+\boldsymbol{H}_{i}^{n+\frac{1}{2}}\right) \times\left(\boldsymbol{E}_{i}^{n+1}+\boldsymbol{E}_{k}^{n+1}\right)\right. \\
& \left.-2\left(\boldsymbol{E}_{i}^{n+1}+\boldsymbol{E}_{i}^{n}\right) \times \boldsymbol{H}_{i}^{n+\frac{1}{2}}\right] \cdot \boldsymbol{n}_{i k} \\
= & -\frac{\tau}{4} \sum_{K \in \nu_{i}} \int_{a_{i k}}\left[\boldsymbol{E}_{i}^{n} \times \boldsymbol{H}_{k}^{n+\frac{1}{2}}-\boldsymbol{E}_{i}^{n} \times \boldsymbol{H}_{i}^{n+\frac{1}{2}}+\boldsymbol{E}_{i}^{n+1} \times \boldsymbol{H}_{i}^{n+\frac{3}{2}}\right. \\
& \left.+\boldsymbol{E}_{k}^{n+1} \times \boldsymbol{H}_{i}^{n+\frac{1}{2}}+\boldsymbol{E}_{i}^{n+1} \times \boldsymbol{H}_{k}^{n+\frac{1}{2}}+\boldsymbol{E}_{k}^{n+1} \times \boldsymbol{H}_{i}^{n+\frac{3}{2}}\right] \cdot \boldsymbol{n}_{i k} .
\end{aligned}
$$

Summing up (36) over all elements and using the jump definition, we have

$$
\sum_{i=1}^{N_{T}} \operatorname{sum}_{1 i}=\frac{\tau}{4} \sum_{i=1}^{N_{T}} \sum_{K \in \nu_{i}} \int_{a_{i k}}\left(\boldsymbol{E}_{i}^{n+1} \times\left[\left[\boldsymbol{H}_{h}^{n+\frac{3}{2}}\right]\right]_{i k}-\boldsymbol{E}_{i}^{n} \times\left[\left[\boldsymbol{H}_{h}^{n+\frac{1}{2}}\right]\right]_{i k}\right) \cdot \boldsymbol{n}_{i k} .
$$

It is easy to see that we have the following identities:

$$
\begin{aligned}
& \frac{\tau}{2} \int_{T_{i}}\left[\nabla \times \boldsymbol{H}_{i}^{n+\frac{1}{2}} \cdot\left(\boldsymbol{E}_{i}^{n+1}+\boldsymbol{E}_{i}^{n}\right)-\boldsymbol{E}_{i}^{n+1} \cdot \nabla \times\left(\boldsymbol{H}_{i}^{n+\frac{3}{2}}+\boldsymbol{H}_{i}^{n+\frac{1}{2}}\right)\right] \\
= & \frac{\tau}{2} \int_{T_{i}}\left[\boldsymbol{E}_{i}^{n} \cdot \nabla \times \boldsymbol{H}_{i}^{n+\frac{1}{2}}-\boldsymbol{E}_{i}^{n+1} \cdot \nabla \times \boldsymbol{H}_{i}^{n+\frac{3}{2}}\right], \\
& \int_{T_{i}} \boldsymbol{E}_{i}^{n+1} \cdot \frac{\tau}{2}\left(\boldsymbol{J}_{i}^{n+\frac{3}{2}}+\boldsymbol{J}_{i}^{n+\frac{1}{2}}\right)-\int_{T_{i}} \boldsymbol{J}_{i}^{n+\frac{1}{2}} \cdot \frac{\tau}{2}\left(\boldsymbol{E}_{i}^{n+1}+\boldsymbol{E}_{i}^{n}\right) \\
= & \frac{\tau}{2} \int_{T_{i}}\left(\boldsymbol{E}_{i}^{n+1} \cdot \boldsymbol{J}_{i}^{n+\frac{3}{2}}-\boldsymbol{E}_{i}^{n} \cdot \boldsymbol{J}_{i}^{n+\frac{1}{2}}\right),
\end{aligned}
$$


and

$$
\begin{aligned}
& \int_{T_{i}} \boldsymbol{H}_{i}^{n+\frac{1}{2}} \cdot \frac{\tau}{2}\left(\boldsymbol{K}_{i}^{n+1}+\boldsymbol{K}_{i}^{n}\right)-\int_{T_{i}} \boldsymbol{K}_{i}^{n+1} \cdot \frac{\tau}{2}\left(\boldsymbol{H}_{i}^{n+\frac{3}{2}}+\boldsymbol{H}_{i}^{n+\frac{1}{2}}\right) \\
= & \frac{\tau}{2} \int_{T_{i}}\left(\boldsymbol{H}_{i}^{n+\frac{1}{2}} \cdot \boldsymbol{K}_{i}^{n}-\boldsymbol{H}_{i}^{n+\frac{3}{2}} \cdot \boldsymbol{K}_{i}^{n+1}\right) .
\end{aligned}
$$

Summing up (34) first over all elements of $\Omega$ and then from $n=0$ to $n=N$, and using the identities (37)-(40), we have

$$
\begin{aligned}
& \frac{\epsilon_{0}}{2}\left(\left\|\boldsymbol{E}_{h}^{N+1}\right\|_{0, \Omega}^{2}-\left\|\boldsymbol{E}_{h}^{0}\right\|_{0, \Omega}^{2}\right)+\frac{\mu_{0}}{2}\left(\left\|\boldsymbol{H}_{h}^{N+\frac{3}{2}}\right\|_{0, \Omega}^{2}-\left\|\boldsymbol{H}_{h}^{\frac{1}{2}}\right\|_{0, \Omega}^{2}\right) \\
& +\frac{1}{2 \epsilon_{0} \omega_{p e}^{2}}\left(\left\|\boldsymbol{J}_{h}^{N+\frac{3}{2}}\right\|_{0, \Omega}^{2}-\left\|\boldsymbol{J}_{h}^{\frac{1}{2}}\right\|_{0, \Omega}^{2}\right)+\frac{1}{2 \mu_{0} \omega_{p m}^{2}}\left(\left\|\boldsymbol{K}_{h}^{N+1}\right\|_{0, \Omega}^{2}-\left\|\boldsymbol{K}_{h}^{0}\right\|_{0, \Omega}^{2}\right) \\
\leq & \frac{\tau}{4} \sum_{i=1}^{N_{T}} \sum_{K \in \nu_{i}} \int_{a_{i k}}\left(\boldsymbol{E}_{i}^{N+1} \times\left[\left[\boldsymbol{H}_{h}^{N+\frac{3}{2}}\right]\right]_{i k}-\boldsymbol{E}_{i}^{0} \times\left[\left[\boldsymbol{H}_{h}^{\frac{1}{2}}\right]\right]_{i k}\right) \cdot \boldsymbol{n}_{i k} \\
+ & \frac{\tau}{2} \int_{\Omega}\left[\boldsymbol{E}_{h}^{0} \cdot \nabla \times \boldsymbol{H}_{h}^{\frac{1}{2}}-\boldsymbol{E}_{h}^{N+1} \cdot \nabla \times \boldsymbol{H}_{h}^{N+\frac{3}{2}}\right] \\
+ & \frac{\tau}{2} \int_{\Omega}\left(\boldsymbol{E}_{h}^{N+1} \cdot \boldsymbol{J}_{h}^{N+\frac{3}{2}}-\boldsymbol{E}_{h}^{0} \cdot \boldsymbol{J}_{h}^{\frac{1}{2}}\right)+\frac{\tau}{2} \int_{\Omega}\left(\boldsymbol{H}_{h}^{\frac{1}{2}} \cdot \boldsymbol{K}_{h}^{0}-\boldsymbol{H}_{h}^{N+\frac{3}{2}} \cdot \boldsymbol{K}_{h}^{N+1}\right) .
\end{aligned}
$$

Now we try to bound the right hand side terms of (41) by the corresponding terms on the left hand side. Below we will constantly use the standard arithmetic-geometric mean (AGM) inequality:

$$
(a, b) \leq \delta\|a\|_{0}^{2}+\frac{1}{4 \delta}\|b\|_{0}^{2}
$$

Using the definition $c_{v}$, the inverse inequalty (30), and the AGM inequality (42), we have

$$
\begin{aligned}
& \frac{\tau}{2} \int_{\Omega} \boldsymbol{E}_{h}^{N+1} \cdot \nabla \times \boldsymbol{H}_{h}^{N+\frac{3}{2}}=\frac{\tau c_{v}}{2} \int_{\Omega} \sqrt{\epsilon_{0}} \boldsymbol{E}_{h}^{N+1} \cdot \sqrt{\mu_{0}} \nabla \times \boldsymbol{H}_{h}^{N+\frac{3}{2}} \\
\leq & \delta_{1} \epsilon_{0}\left\|\boldsymbol{E}_{h}^{N+1}\right\|_{0, \Omega}^{2}+\frac{1}{4 \delta_{1}} \cdot\left(\frac{\tau c_{v} c_{i n v} h^{-1}}{2}\right)^{2} \cdot \mu_{0}\left\|\boldsymbol{H}_{h}^{N+\frac{3}{2}}\right\|_{0, \Omega}^{2} .
\end{aligned}
$$

Similarly, we can obtain

$$
\begin{aligned}
& \frac{\tau}{2} \int_{\Omega} \boldsymbol{E}_{h}^{N+1} \cdot \boldsymbol{J}_{h}^{N+\frac{3}{2}}=\frac{\tau \omega_{p e}}{2} \int_{\Omega} \sqrt{\epsilon_{0}} \boldsymbol{E}_{h}^{N+1} \cdot \frac{1}{\sqrt{\epsilon_{0}} \omega_{p e}} \boldsymbol{J}_{h}^{N+\frac{3}{2}} \\
\leq & \delta_{2} \epsilon_{0}\left\|\boldsymbol{E}_{h}^{N+1}\right\|_{0, \Omega}^{2}+\frac{1}{4 \delta_{2}} \cdot\left(\frac{\tau \omega_{p e}}{2}\right)^{2} \cdot \frac{1}{\epsilon_{0} \omega_{p e}^{2}}\left\|\boldsymbol{J}_{h}^{N+\frac{3}{2}}\right\|_{0, \Omega}^{2},
\end{aligned}
$$

and

$$
\begin{aligned}
& \frac{\tau}{2} \int_{\Omega} \boldsymbol{H}_{h}^{N+\frac{3}{2}} \cdot \boldsymbol{K}_{h}^{N+1}=\frac{\tau \omega_{p m}}{2} \int_{\Omega} \sqrt{\mu_{0}} \boldsymbol{H}_{h}^{N+\frac{3}{2}} \cdot \frac{1}{\sqrt{\mu_{0}} \omega_{p m}} \boldsymbol{K}_{h}^{N+1} \\
\leq & \delta_{3} \mu_{0}\left\|\boldsymbol{H}_{h}^{N+\frac{3}{2}}\right\|_{0, \Omega}^{2}+\frac{1}{4 \delta_{3}} \cdot\left(\frac{\tau \omega_{p m}}{2}\right)^{2} \cdot \frac{1}{\mu_{0} \omega_{p m}^{2}}\left\|\boldsymbol{K}_{h}^{N+1}\right\|_{0, \Omega}^{2} .
\end{aligned}
$$


Finally, using the trace inequality (31) and the AGM inequality (42), we have

$$
\begin{aligned}
& \frac{\tau}{4} \sum_{i=1}^{N_{T}} \sum_{K \in \nu_{i}} \int_{a_{i k}}\left(\boldsymbol{E}_{i}^{N+1} \times\left[\left[\boldsymbol{H}_{h}^{N+\frac{3}{2}}\right]\right]_{i k}\right) \cdot \boldsymbol{n}_{i k} \\
\leq & \frac{\tau}{4} \sum_{i=1}^{N_{T}} c_{t r} h^{-1 / 2}\left\|\boldsymbol{E}_{i}^{N+1}\right\|_{0, T_{i}} \cdot c_{t r} h^{-1 / 2}\left\|\boldsymbol{H}_{h}^{N+\frac{3}{2}}\right\|_{0, T_{i}} \\
= & \frac{\tau}{4} \cdot c_{t r}^{2} h^{-1} c_{v} \sum_{i=1}^{N_{T}} \sqrt{\epsilon_{0}}\left\|\boldsymbol{E}_{i}^{N+1}\right\|_{0, T_{i}} \cdot \sqrt{\mu_{0}}\left\|\boldsymbol{H}_{h}^{N+\frac{3}{2}}\right\|_{0, T_{i}} \\
\leq & \delta_{4} \epsilon\left\|\boldsymbol{E}_{h}^{N+1}\right\|_{0, \Omega}^{2}+\frac{1}{4 \delta_{4}} \cdot\left(\frac{\tau c_{t r}^{2} c_{v} h^{-1}}{4}\right)^{2} \cdot \mu_{0}\left\|\boldsymbol{H}_{h}^{N+\frac{3}{2}}\right\|_{0, \Omega}^{2} .
\end{aligned}
$$

First choosing $\delta_{i}(i=1,2,3,4)$ small enough, then choosing $\tau$ satisfying the CFL condition $\tau \leq C h$, we can obtain the following stability

$$
\begin{aligned}
& \epsilon_{0}\left\|\boldsymbol{E}_{h}^{N+1}\right\|_{0, \Omega}^{2}+\mu_{0}\left\|\boldsymbol{H}_{h}^{N+\frac{3}{2}}\right\|_{0, \Omega}^{2}+\frac{1}{\epsilon_{0} \omega_{p e}^{2}}\left\|\boldsymbol{J}_{h}^{N+\frac{3}{2}}\right\|_{0, \Omega}^{2}+\frac{1}{\mu_{0} \omega_{p m}^{2}}\left\|\boldsymbol{K}_{h}^{N+1}\right\|_{0, \Omega}^{2} \\
\leq & C\left[\epsilon_{0}\left\|\boldsymbol{E}_{h}^{0}\right\|_{0, \Omega}^{2}+\mu_{0}\left\|\boldsymbol{H}_{h}^{\frac{1}{2}}\right\|_{0, \Omega}^{2}+\frac{1}{\epsilon_{0} \omega_{p e}^{2}}\left\|\boldsymbol{J}_{h}^{\frac{1}{2}}\right\|_{0, \Omega}^{2}+\frac{1}{\mu_{0} \omega_{p m}^{2}}\left\|\boldsymbol{K}_{h}^{0}\right\|_{0, \Omega}^{2}\right] .
\end{aligned}
$$

A simple choice is $\delta_{1}=\delta_{2}=\delta_{3}=\delta_{4}=\frac{1}{8}$, and

$$
\left(\tau c_{v} c_{i n v} h^{-1}\right)^{2} \leq \frac{1}{4}, \quad\left(\tau c_{t r}^{2} c_{v} h^{-1}\right)^{2} \leq 1, \quad\left(\tau \omega_{p e}\right)^{2} \leq \frac{1}{2}, \quad\left(\tau \omega_{p m}\right)^{2} \leq \frac{1}{2},
$$

which leads to a choice of $\tau$ as (32). This completes our proof. $\square$

Note that the above stability result is obtained without using the discrete Gronwall inequality often seen in analyzing time-dependent problems. Finally, we like to point out the following error estimate for our scheme (26)-(29).

Theorem 2.4 Let $\left(\boldsymbol{E}^{n+1}, \boldsymbol{H}^{n+\frac{3}{2}}, \boldsymbol{J}^{n+\frac{3}{2}}, \boldsymbol{K}^{n+1}\right)$ and $\left(\boldsymbol{E}_{h}^{n+1}, \boldsymbol{H}_{h}^{n+\frac{3}{2}}, \boldsymbol{J}_{h}^{n+\frac{3}{2}}, \boldsymbol{K}_{h}^{n+1}\right)$ be the solutions of (1)-(4) and (26)-(29) at time $t_{n+1}$ or $t_{n+\frac{3}{2}}$. Then under the CFL condition (32), there exists a constant $C>0$, independent of $\tau$ and $h$, such that for any $n \geq 0$ we have

$$
\begin{aligned}
& \max _{n \geq 0}\left(\left\|\boldsymbol{E}_{h}^{n+1}-\boldsymbol{E}^{n+1}\right\|_{0, \Omega}+\left\|\boldsymbol{H}_{h}^{n+\frac{3}{2}}-\boldsymbol{H}^{n+\frac{3}{2}}\right\|_{0, \Omega}+\left\|\boldsymbol{J}_{h}^{n+\frac{3}{2}}-\boldsymbol{J}^{n+\frac{3}{2}}\right\|_{0, \Omega}+\left\|\boldsymbol{K}_{h}^{n+1}-\boldsymbol{K}^{n+1}\right\|_{0, \Omega}\right) \\
\leq & C\left(\tau^{2}+h^{k}\right)+C\left(\left\|\boldsymbol{E}_{h}^{0}-\boldsymbol{E}^{0}\right\|_{0, \Omega}+\left\|\boldsymbol{H}_{h}^{\frac{1}{2}}-\boldsymbol{H}^{\frac{1}{2}}\right\|_{0, \Omega}+\left\|\boldsymbol{J}_{h}^{\frac{1}{2}}-\boldsymbol{J}^{\frac{1}{2}}\right\|_{0, \Omega}+\left\|\boldsymbol{K}_{h}^{0}-\boldsymbol{K}^{0}\right\|_{0, \Omega}\right), \quad(47)
\end{aligned}
$$

where $k \geq 1$ is the order of basis function in the space $\boldsymbol{V}_{h}$.

Proof. Integrating (8) and (11) from $t_{n}$ to $t_{n+1}$, and (9) and (10) from $t_{n+\frac{1}{2}}$ to $t_{n+\frac{3}{2}}$, we have

$$
\begin{aligned}
& \epsilon_{0} \int_{T_{i}} \frac{\boldsymbol{E}^{n+1}-\boldsymbol{E}^{n}}{\tau} \cdot \boldsymbol{u}-\int_{T_{i}}\left(\frac{1}{\tau} \int_{t_{n}}^{t_{n+1}} \boldsymbol{H}\right) \cdot \nabla \times \boldsymbol{u}-\int_{\partial T_{i}} \boldsymbol{n}_{i} \times\left(\frac{1}{\tau} \int_{t_{n}}^{t_{n+1}} \boldsymbol{H}\right) \cdot \boldsymbol{u}+\int_{T_{i}}\left(\frac{1}{\tau} \int_{t_{n}}^{t_{n+1}} \boldsymbol{J}\right) \cdot \boldsymbol{u}=0, \\
& \mu_{0} \int_{T_{i}} \frac{\boldsymbol{H}^{n+\frac{3}{2}}-\boldsymbol{H}^{n+\frac{1}{2}}}{\tau} \cdot \boldsymbol{v}+\int_{T_{i}}\left(\frac{1}{\tau} \int_{t_{n+\frac{1}{2}}}^{t_{n+\frac{3}{2}}} \boldsymbol{E}\right) \cdot \nabla \times \boldsymbol{v}+\int_{\partial T_{i}} \boldsymbol{n}_{i} \times\left(\frac{1}{\tau} \int_{t_{n+\frac{1}{2}}}^{t_{n+\frac{3}{2}}} \boldsymbol{E}\right) \cdot \boldsymbol{v}+\int_{T_{i}}\left(\frac{1}{\tau} \int_{t_{n+\frac{1}{2}}}^{t_{n+\frac{3}{2}}} \boldsymbol{K}\right) \cdot \boldsymbol{v}=0,
\end{aligned}
$$




$$
\begin{aligned}
& \frac{1}{\epsilon_{0} \omega_{p e}^{2}} \int_{T_{i}} \frac{\boldsymbol{J}^{n+\frac{3}{2}}-\boldsymbol{J}^{n+\frac{1}{2}}}{\tau} \cdot \boldsymbol{\phi}+\frac{\Gamma_{e}}{\epsilon_{0} \omega_{p e}^{2}} \int_{T_{i}}\left(\frac{1}{\tau} \int_{t_{n+\frac{1}{2}}}^{t_{n+\frac{3}{2}}} \boldsymbol{J}\right) \cdot \boldsymbol{\phi}=\int_{T_{i}}\left(\frac{1}{\tau} \int_{t_{n+\frac{1}{2}}}^{t_{n+\frac{3}{2}}} \boldsymbol{E}\right) \cdot \boldsymbol{\phi}, \\
& \frac{1}{\mu_{0} \omega_{p m}^{2}} \int_{T_{i}} \frac{\boldsymbol{K}^{n+1}-\boldsymbol{K}^{n}}{\tau} \cdot \boldsymbol{\psi}+\frac{\Gamma_{m}}{\mu_{0} \omega_{p m}^{2}} \int_{T_{i}}\left(\frac{1}{\tau} \int_{t_{n}}^{t_{n+1}} \boldsymbol{K}\right) \cdot \boldsymbol{\psi}=\int_{T_{i}}\left(\frac{1}{\tau} \int_{t_{n}}^{t_{n+1}} \boldsymbol{H}\right) \cdot \boldsymbol{\psi} .
\end{aligned}
$$

Then we obtain error equations by subtracting (48)-(50) from (26)-(29). The rest of the proof is similar to the stability proof carried out for Theorem 2.3 , and is skipped due to the technicality.

\section{Extension of the DG method to the PML model}

To model wave propagation in practice, we need to truncate an infinite physical domain to a bounded domain. To easily couple with our metamaterial Maxwell's equations (1)-(4), we choose a perfectly matched layer (PML) model developed by Ziolkowski [20] in 1997. Following [20], the PML is assumed to be a cubical simulation domain, and the complete PML governing equations for the corner region are [20, Eq. (B.4)]:

$$
\begin{aligned}
& \frac{\partial \boldsymbol{E}}{\partial t}+D_{1} \boldsymbol{E}=\frac{1}{\epsilon_{0}} \nabla \times \boldsymbol{H}-\frac{1}{\epsilon_{0}} \boldsymbol{J}, \\
& \frac{\partial \boldsymbol{J}}{\partial t}+D_{2} \boldsymbol{J}=\epsilon_{0} D_{3} \boldsymbol{E}, \\
& \frac{\partial \boldsymbol{H}}{\partial t}+D_{1} \boldsymbol{H}=-\frac{1}{\mu_{0}} \nabla \times \boldsymbol{E}-\frac{1}{\mu_{0}} \boldsymbol{K}, \\
& \frac{\partial \boldsymbol{K}}{\partial t}+D_{2} \boldsymbol{K}=\mu_{0} D_{3} \boldsymbol{H},
\end{aligned}
$$

where $\epsilon_{0}$ and $\mu_{0}$ are the vacuum permittivity and permeability, $\boldsymbol{E}(\boldsymbol{x}, t)$ and $\boldsymbol{H}(\boldsymbol{x}, t)$ are the electric and magnetic fields, $\boldsymbol{J}(\boldsymbol{x}, t)$ and $\boldsymbol{K}(\boldsymbol{x}, t)$ are the induced electric and magnetic currents, respectively. Furthermore, the $3 \times 3$ diagonal matrices $D_{1}=\operatorname{diag}\left(\sigma_{y}+\sigma_{z}-\sigma_{x}, \sigma_{z}+\sigma_{x}-\sigma_{y}, \sigma_{x}+\sigma_{y}-\sigma_{z}\right)$, $D_{2}=\operatorname{diag}\left(\sigma_{x}, \sigma_{y}, \sigma_{z}\right), D_{3}=\operatorname{diag}\left(\left(\sigma_{x}-\sigma_{y}\right)\left(\sigma_{x}-\sigma_{z}\right),\left(\sigma_{y}-\sigma_{x}\right)\left(\sigma_{y}-\sigma_{z}\right),\left(\sigma_{z}-\sigma_{x}\right)\left(\sigma_{z}-\sigma_{y}\right)\right)$. Here $\sigma_{x}, \sigma_{y}$ and $\sigma_{z}$ are nonnegative functions and represent the damping variations along the $x, y$ and $z$ directions, respectively. Note that the PML equations (51)-(54) should be modified accordingly at other regions. For example, in the face regions, only one normal direction has absorbing layers.

Note that the model (51)-(54) is the same as (5.12) of Turkel and Yefet [18] (with assumption $\epsilon_{0}=\mu_{0}=1$ ) and is well-posed mathematically because it is a symmetric hyperbolic system (i.e., the standard Maxwell equations) plus lower order terms [18, p.545].

We like to remark that all the results in previous section can be extended easily to this PML model, but the proofs become more involved. For clarity, below we just briefly present the semiand fully-discrete schemes for solving (51)-(54).

The semi-discrete DG method for (51)-(54): For any $\boldsymbol{u}_{h}, \boldsymbol{v}_{h}, \boldsymbol{\phi}_{h}, \boldsymbol{\psi}_{h} \in \boldsymbol{V}_{h}$, and any element $T_{i} \in T_{h}$, find $\boldsymbol{E}_{h}, \boldsymbol{H}_{h}, \boldsymbol{J}_{h}, \boldsymbol{K}_{h} \in C^{1}\left(0, T ; \boldsymbol{V}_{h}\right)$ such that

$$
\begin{aligned}
& \epsilon_{0} \int_{T_{i}} \frac{\partial \boldsymbol{E}_{h}}{\partial t} \cdot \boldsymbol{u}_{h}-\int_{T_{i}} \boldsymbol{H}_{h} \cdot \nabla \times \boldsymbol{u}_{h}-\sum_{K \in \nu_{i}} \int_{a_{i k}} \boldsymbol{u}_{h} \cdot \boldsymbol{n}_{i k} \times\left\{\left\{\boldsymbol{H}_{h}\right\}\right\}_{i k}+\int_{T_{i}}\left(\boldsymbol{J}_{h}+\epsilon_{0} D_{1} \boldsymbol{E}_{h}\right) \cdot \boldsymbol{u}_{h}=0, \\
& \mu_{0} \int_{T_{i}} \frac{\partial \boldsymbol{H}_{h}}{\partial t} \cdot \boldsymbol{v}_{h}+\int_{T_{i}} \boldsymbol{E}_{h} \cdot \nabla \times \boldsymbol{v}_{h}+\sum_{K \in \nu_{i}} \int_{a_{i k}} \boldsymbol{v}_{h} \cdot \boldsymbol{n}_{i k} \times\left\{\left\{\boldsymbol{E}_{h}\right\}\right\}_{i k}+\int_{T_{i}}\left(\boldsymbol{K}_{h}+\mu_{0} D_{1} \boldsymbol{H}_{h}\right) \cdot \boldsymbol{v}_{h}=0,
\end{aligned}
$$




$$
\begin{aligned}
& \frac{1}{\epsilon_{0}} \int_{T_{i}} \frac{\partial \boldsymbol{J}_{h}}{\partial t} \cdot \boldsymbol{\phi}_{h}+\frac{1}{\epsilon_{0}} \int_{T_{i}} D_{2} \boldsymbol{J}_{h} \cdot \boldsymbol{\phi}_{h}=\int_{T_{i}} D_{3} \boldsymbol{E}_{h} \cdot \boldsymbol{\phi}_{h}, \\
& \frac{1}{\mu_{0}} \int_{T_{i}} \frac{\partial \boldsymbol{K}_{h}}{\partial t} \cdot \boldsymbol{\psi}_{h}+\frac{1}{\mu_{0}} \int_{T_{i}} D_{2} \boldsymbol{K}_{h} \cdot \boldsymbol{\psi}_{h}=\int_{T_{i}} D_{3} \boldsymbol{H}_{h} \cdot \boldsymbol{\psi}_{h},
\end{aligned}
$$

hold true and are subject to the initial conditions (16).

Denote the semi-discrete energy $\mathcal{E}_{h}^{p m l}$ :

$$
\mathcal{E}_{h}^{p m l}(t)=\frac{1}{2}\left(\epsilon_{0}\left\|\boldsymbol{E}_{h}(t)\right\|_{0, \Omega}^{2}+\mu_{0}\left\|\boldsymbol{H}_{h}(t)\right\|_{0, \Omega}^{2}+\frac{1}{\epsilon_{0}}\left\|\boldsymbol{J}_{h}(t)\right\|_{0, \Omega}^{2}+\frac{1}{\mu_{0}}\left\|\boldsymbol{K}_{h}(t)\right\|_{0, \Omega}^{2}\right) .
$$

Theorem 3.1 The energy $\mathcal{E}_{h}^{\text {pml }}$ is bounded, i.e., $\mathcal{E}_{h}^{\text {pml }}(t) \leq C \mathcal{E}_{h}^{p m l}(0)$ holds true for any $t \in[0, T]$, where the constant $T>0$ depends on $T$.

Proof. Choosing $\boldsymbol{u}_{h}=\boldsymbol{E}_{h}, \boldsymbol{v}_{h}=\boldsymbol{H}_{h}, \boldsymbol{\phi}_{h}=\boldsymbol{J}_{h}, \boldsymbol{\psi}_{h}=\boldsymbol{K}_{h}$ in (55)-(58) and adding the results together over all element $T_{i} \in T_{h}$, we obtain

$$
\begin{aligned}
& \frac{d}{d t} \mathcal{E}_{h}^{p m l}(t)+\left\|\sqrt{\frac{D_{2}}{\epsilon_{0}}} \boldsymbol{J}_{h}(t)\right\|_{0, \Omega}^{2}+\left\|\sqrt{\frac{D_{2}}{\mu_{0}}} \boldsymbol{K}_{h}(t)\right\|_{0, \Omega}^{2}+\sum_{i} \mathcal{B}_{i}\left(\boldsymbol{E}_{h}, \boldsymbol{H}_{h}\right) \\
= & -\int_{\Omega} \boldsymbol{J}_{h} \cdot \boldsymbol{E}_{h}-\int_{\Omega} \epsilon_{0} D_{1} \boldsymbol{E}_{h} \cdot \boldsymbol{E}_{h}-\int_{\Omega} \boldsymbol{K}_{h} \cdot \boldsymbol{H}_{h} \\
& -\int_{\Omega} \mu_{0} D_{1} \boldsymbol{H}_{h} \cdot \boldsymbol{H}_{h}+\int_{\Omega} D_{3} \boldsymbol{E}_{h} \cdot \boldsymbol{J}_{h}+\int_{\Omega} D_{3} \boldsymbol{H}_{h} \cdot \boldsymbol{K}_{h} \\
= & \int_{\Omega}\left(D_{3}-I_{3}\right) \boldsymbol{H}_{h} \cdot \boldsymbol{K}_{h}+\int_{\Omega}\left(D_{3}-I_{3}\right) \boldsymbol{E}_{h} \cdot \boldsymbol{J}_{h}-\int_{\Omega} \epsilon_{0} D_{1} \boldsymbol{E}_{h} \cdot \boldsymbol{E}_{h}-\int_{\Omega} \mu_{0} D_{1} \boldsymbol{H}_{h} \cdot \boldsymbol{H}_{h},
\end{aligned}
$$

where we denote $I_{3}$ for the $3 \times 3$ identity matrix.

Using the fact that $\sum_{i} \mathcal{B}_{i}\left(\boldsymbol{E}_{h}, \boldsymbol{H}_{h}\right)=0$, the Cauchy-Schwarz inequality to those right hand side terms of (60) with the boundness of $D_{1}$ and $D_{3}$, and the Gronwall inequality, we complete the proof.

Similar to the metamaterial model, we can construct the following leap-frog DG scheme: Given initial approximations of $\boldsymbol{E}_{h}^{0}, \boldsymbol{H}_{h}^{\frac{1}{2}}, \boldsymbol{J}_{h}^{\frac{1}{2}}, \boldsymbol{K}_{h}^{0}$, for any $n \geq 0$, find $\boldsymbol{E}_{h}^{n+1}, \boldsymbol{H}_{h}^{n+\frac{3}{2}}, \boldsymbol{J}_{h}^{n+\frac{3}{2}}, \boldsymbol{K}_{h}^{n+1} \in \boldsymbol{V}_{h}$ such that

$$
\begin{aligned}
& \epsilon_{0} \int_{T_{i}} \frac{\boldsymbol{E}_{h}^{n+1}-\boldsymbol{E}_{h}^{n}}{\tau} \cdot \boldsymbol{u}_{h}-\int_{T_{i}} \boldsymbol{H}_{h}^{n+\frac{1}{2}} \cdot \nabla \times \boldsymbol{u}_{h}-\sum_{K \in \nu_{i}} \int_{a_{i k}} \boldsymbol{u}_{h} \cdot \boldsymbol{n}_{i k} \times\left\{\left\{\boldsymbol{H}_{h}^{n+\frac{1}{2}}\right\}\right\}_{i k} \\
& \quad+\int_{T_{i}}\left(\boldsymbol{J}_{h}^{n+\frac{1}{2}}+\frac{\epsilon_{0} D_{1}}{2}\left(\boldsymbol{E}_{h}^{n+1}+\boldsymbol{E}_{h}^{n}\right)\right) \cdot \boldsymbol{u}_{h}=0 \\
& \mu_{0} \int_{T_{i}} \frac{\boldsymbol{H}_{h}^{n+\frac{3}{2}}-\boldsymbol{H}_{h}^{n+\frac{1}{2}}}{\tau} \cdot \boldsymbol{v}_{h}+\int_{T_{i}} \boldsymbol{E}_{h}^{n+1} \cdot \nabla \times \boldsymbol{v}_{h}+\sum_{K \in \nu_{i}} \int_{a_{i k}} \boldsymbol{v}_{h} \cdot \boldsymbol{n}_{i k} \times\left\{\left\{\boldsymbol{E}_{h}^{n+1}\right\}\right\}_{i k} \\
& \quad+\int_{T_{i}}\left(\boldsymbol{K}_{h}^{n+1}+\frac{\mu_{0} D_{1}}{2}\left(\boldsymbol{H}_{h}^{n+\frac{3}{2}}+\boldsymbol{H}_{h}^{n+\frac{1}{2}}\right)\right) \cdot \boldsymbol{v}_{h}=0, \\
& \frac{1}{\epsilon_{0}} \int_{T_{i}} \frac{\boldsymbol{J}_{h}^{n+\frac{3}{2}}-\boldsymbol{J}_{h}^{n+\frac{1}{2}}}{\tau} \cdot \boldsymbol{\phi}_{h}+\int_{T_{i}} \frac{D_{2}}{2 \epsilon_{0}}\left(\boldsymbol{J}_{h}^{n+\frac{3}{2}}+\boldsymbol{J}_{h}^{n+\frac{1}{2}}\right) \cdot \boldsymbol{\phi}_{h}=\int_{T_{i}} D_{3} \boldsymbol{E}_{h}^{n+1} \cdot \boldsymbol{\phi}_{h}, \\
& \frac{1}{\mu_{0}} \int_{T_{i}} \frac{\boldsymbol{K}_{h}^{n+1}-\boldsymbol{K}_{h}^{n}}{\tau} \cdot \boldsymbol{\psi}_{h}+\int_{T_{i}} \frac{D_{2}}{2 \mu_{0}}\left(\boldsymbol{K}_{h}^{n+1}+\boldsymbol{K}_{h}^{n}\right) \cdot \boldsymbol{\psi}_{h}=\int_{T_{i}} D_{3} \boldsymbol{H}_{h}^{n+\frac{1}{2}} \cdot \boldsymbol{\psi}_{h},
\end{aligned}
$$


hold true for any $\boldsymbol{u}_{h}, \boldsymbol{v}_{h}, \boldsymbol{\phi}_{h}, \boldsymbol{\psi}_{h} \in \boldsymbol{V}_{h}$, and any element $T_{i} \in T_{h}$.

In implementation, at each time step, we first solve (61) and (64) in parallel; then we solve (62) and (63) simultaneously.

\section{$4 \quad$ Numerical results}

Our implementation is based on the package nudg provided by [8]. The theoretical results hold true for both 3-D and 2-D cases. Here we show some interesting 2-D numerical results. All our tests are carried out using MATLAB R2011b running on Dell Precision WorkStation T7500 with 12GB memory and $2.26 \mathrm{GHz}$ Intel Xeon CPU.

\subsection{Convergence rate test for the metamaterial model}

Here we consider the 2-D transverse magnetic metamaterial model:

$$
\begin{aligned}
\frac{\partial H_{x}}{\partial t} & =-\frac{\partial E_{z}}{\partial y}-K_{x}+g_{x} \\
\frac{\partial H_{y}}{\partial t} & =\frac{\partial E_{z}}{\partial x}-K_{y}+g_{y} \\
\frac{\partial E_{z}}{\partial t} & =\frac{\partial H_{y}}{\partial x}-\frac{\partial H_{x}}{\partial y}-J_{z}+f, \\
\frac{\partial J_{z}}{\partial t} & =\omega_{e}^{2} E_{z}-\Gamma_{e} J_{z} \\
\frac{\partial K_{x}}{\partial t} & =\omega_{m}^{2} H_{x}-\Gamma_{m} K_{x} \\
\frac{\partial K_{y}}{\partial t} & =\omega_{m}^{2} H_{y}-\Gamma_{m} K_{y}
\end{aligned}
$$

which is the 2-D version of (1)-(4) with $\epsilon_{0}=\mu_{0}=1$ and added source terms $g_{x}, g_{y}$ and $f$. In (65)-(70) the subscripts ' $x, y$ ' and ' $z$ ' denote the corresponding components.

Example 1. To check the convergence rate for our scheme, we use the same exact solutions constructed in our previous work [11] (assuming that $\Gamma_{m}=\Gamma_{e}=\pi, \omega_{m}=\omega_{e}=\pi$ ) on domain $\Omega=(0,1)^{2}$ :

$$
\begin{gathered}
\boldsymbol{H} \equiv\left(\begin{array}{c}
H_{x} \\
H_{y}
\end{array}\right)=\left(\begin{array}{c}
\sin (\pi x) \cos (\pi y) \exp (-\pi t) \\
-\cos (\pi x) \sin (\pi y) \exp (-\pi t)
\end{array}\right), \\
E_{z}=\sin (\pi x) \sin (\pi y) \exp (-\pi t) .
\end{gathered}
$$

The corresponding magnetic and electric currents are

$$
\boldsymbol{K} \equiv\left(\begin{array}{c}
K_{x} \\
K_{y}
\end{array}\right)=\left(\begin{array}{c}
\pi^{2} t \sin (\pi x) \cos (\pi y) \exp (-\pi t) \\
-\pi^{2} t \cos (\pi x) \sin (\pi y) \exp (-\pi t)
\end{array}\right)
$$

and

$$
J_{z}=\pi^{2} t \sin (\pi x) \sin (\pi y) \exp (-\pi t),
$$

respectively. The corresponding source term

$$
f=\left(-3 \pi+\pi^{2} t\right) \sin (\pi x) \sin (\pi y) \exp (-\pi t),
$$


Table 1: Example 1. $L_{\infty}$ and $L_{2}$ errors for magnetic field $H_{x}$ with linear basis function and $\tau=10^{-5}$ at 1000 time steps

\begin{tabular}{|c|c|c|c|c|c|c|c|c|c|}
\hline & $h=1 / 5$ & $h=1 / 10$ & Rates & $h=1 / 20$ & Rates & $h=1 / 40$ & Rates & $h=1 / 80$ & Rates \\
\hline$L_{\infty}$ errors & 0.0171 & 0.0095 & 0.8480 & 0.0048 & 0.9849 & 0.0024 & 1.0 & 0.0012 & 1.0 \\
$L_{2}$ errors & 0.0046 & 0.0023 & 1.0 & 0.0011 & 1.0641 & $5.2752 \mathrm{e}-4$ & 1.0602 & $2.1700 \mathrm{e}-4$ & 1.2815 \\
CPU(s) & 17.19 & 17.65 & & 29.61 & & 33.72 & & 44.05 & \\
\hline
\end{tabular}

Table 2: Example 1. $L_{\infty}$ and $L_{2}$ errors for magnetic field $H_{x}$ with quadratic basis function and $\tau=10^{-5}$ at 1000 time steps

\begin{tabular}{|c|c|c|c|c|c|c|c|c|c|}
\hline & $h=1 / 5$ & $h=1 / 10$ & Rates & $h=1 / 20$ & Rates & $h=1 / 40$ & Rates & $h=1 / 80$ & Rates \\
\hline$L_{\infty}$ errors & 0.0039 & $9.7298 \mathrm{e}-4$ & 2.0030 & $2.1093 \mathrm{e}-4$ & 2.2056 & $4.7594 \mathrm{e}-5$ & 2.1479 & $2.2728 \mathrm{e}-5$ & 1.0663 \\
$L_{2}$ errors & $5.6795 \mathrm{e}-4$ & $1.4231 \mathrm{e}-4$ & 1.9967 & $3.3759 \mathrm{e}-5$ & 2.0757 & $9.9027 \mathrm{e}-6$ & 1.7694 & $7.6578 \mathrm{e}-6$ & 0.3709 \\
CPU(s) & 18.76 & 19.60 & & 30.38 & & 33.48 & & 58.35 & \\
\hline
\end{tabular}

while $\boldsymbol{g}=\left(g_{x}, g_{y}\right)$ is given by

$$
\begin{aligned}
& g_{x}=\pi^{2} t \sin (\pi x) \cos (\pi y) \exp (-\pi t), \\
& g_{y}=-\pi^{2} t \cos (\pi x) \sin (\pi y) \exp (-\pi t),
\end{aligned}
$$

Notice that $E_{z}$ satisfies the boundary condition $E_{z}=0$ on $\partial \Omega$.

We solved this problem with various time steps $\tau$ and uniformly refined triangular meshes. In Table 1, we presented the numerical results obtained with linear basis function, $\tau=10^{-5}$ running for 1000 time steps. Since the convergence rates are very similar for all the variables, we just presented $H_{x}$ in Table 1, which shows clearly $O(h)$ rates in both $L_{\infty}$ and $L_{2}$ norms.

In Table 2, we presented the numerical results obtained with quadratic basis function, $\tau=10^{-5}$ running for 1000 time steps. Results of Table 2 show $O\left(h^{2}\right)$ rates in both $L_{\infty}$ and $L_{2}$ norms, though the rates degenerate as the mesh becomes fine enough. The reason is that the solution error is dominated by the time error when the mesh is fine enough.

Example 2. To further check the convergence rate and our algorithmic implementation, we construct another exact solution for (65)-(70) on $\Omega=(0,1)^{2}$ by assuming that $\Gamma_{m}=\Gamma_{e}=2 \omega \pi, \omega_{m}=$ $\omega_{e}=\omega \pi$ :

$$
\begin{aligned}
\boldsymbol{H} & \equiv\left(\begin{array}{c}
H_{x} \\
H_{y}
\end{array}\right)=\left(\begin{array}{c}
\sin (\omega \pi x) \cos (\omega \pi y) \exp (-\omega \pi t) \\
-\cos (\omega \pi x) \sin (\omega \pi y) \exp (-\omega \pi t)
\end{array}\right), \\
E_{z} & =\sin (\omega \pi x) \sin (\omega \pi y) \exp (-\omega \pi t), \\
\boldsymbol{K} & \equiv\left(\begin{array}{c}
K_{x} \\
K_{y}
\end{array}\right)=\omega \pi\left(\begin{array}{c}
\sin (\omega \pi x) \cos (\omega \pi y) \exp (-\omega \pi t) \\
-\cos (\omega \pi x) \sin (\omega \pi y) \exp (-\omega \pi t)
\end{array}\right), \\
J_{z} & =\omega \pi \sin (\omega \pi x) \sin (\omega \pi y) \exp (-\omega \pi t),
\end{aligned}
$$

which leads to source terms

$$
\begin{aligned}
f & =-2 \omega \pi \sin (\omega \pi x) \sin (\omega \pi y) \exp (-\omega \pi t) \\
g_{x} & =K_{x}, \quad g_{y}=K_{y} .
\end{aligned}
$$


Table 3: Example 2. $L_{\infty}$ and $L_{2}$ errors for magnetic field $H_{x}$ with linear basis function and $\tau=10^{-8}$ at 1000 time steps

\begin{tabular}{|c|c|c|c|c|c|c|c|c|c|}
\hline & $h=1 / 5$ & $h=1 / 10$ & Rates & $h=1 / 20$ & Rates & $h=1 / 40$ & Rates & $h=1 / 80$ & Rates \\
\hline$L_{\infty}$ errors & $1.6979 \mathrm{e}-4$ & $1.2971 \mathrm{e}-4$ & 0.3885 & $7.3808 \mathrm{e}-5$ & 0.8134 & $3.8803 \mathrm{e}-5$ & 0.9276 & $1.9643 \mathrm{e}-5$ & 0.9822 \\
$L_{2}$ errors & $5.3631 \mathrm{e}-5$ & $3.6010 \mathrm{e}-5$ & 0.5747 & $1.9377 \mathrm{e}-5$ & 0.8941 & $9.8288 \mathrm{e}-6$ & 0.9793 & $4.9160 \mathrm{e}-6$ & 0.9995 \\
\hline
\end{tabular}

Table 4: Example 2. $L_{\infty}$ and $L_{2}$ errors for magnetic field $H_{x}$ with quadratic basis function and $\tau=10^{-8}$ at 1000 time steps.

\begin{tabular}{|c|c|c|c|c|c|c|c|c|c|}
\hline & $h=1 / 5$ & $h=1 / 10$ & Rates & $h=1 / 20$ & Rates & $h=1 / 40$ & Rates & $h=1 / 80$ & Rates \\
\hline$L_{\infty}$ errors & $1.7836 \mathrm{e}-4$ & $5.9329 \mathrm{e}-5$ & 1.5880 & $1.5958 \mathrm{e}-5$ & 1.8945 & $4.1534 \mathrm{e}-6$ & 1.9419 & $1.0931 \mathrm{e}-6$ & 1.9259 \\
$L_{2}$ errors & $2.9229 \mathrm{e}-5$ & $9.1135 \mathrm{e}-6$ & 1.6813 & $2.3892 \mathrm{e}-6$ & 1.9315 & $6.0817 \mathrm{e}-7$ & 1.9740 & $1.5630 \mathrm{e}-7$ & 1.9602 \\
\hline
\end{tabular}

Many numerical tests have been carried out for various $h, \tau$ and $\omega$. Selected results are presented for $\omega=4$ in Tables 3-4, which again justify the theoretical convergence rate $O\left(\tau^{2}+h^{r}\right)$ for a r-th order basis function when $r \leq 2$. When $r \geq 3$, we need smaller time steps in order to see the spatial convergence rate clearly, since the error will be saturated when the mesh is fine enough. In Tables 5-6, we presented the results obtained with $\omega=4, r=3, \tau=10^{-12}$ at 1 and 1000 time steps, respectively. Tables 5-6 show the convergence rate $O\left(h^{3}\right)$ clearly.

Finally, we present a test for checking the time convergence rate $O\left(\tau^{2}\right)$. Since the time step depends on $h$, we can not check this using the traditional way by fixing a very small $h$ with various $\tau$. Here we solve the problem with quadratic basis function and $\omega=4$. We fix $\tau=0.01 h$, and the final time $T=0.1$ (i.e, the number of time step varies as $n t=10 / h$ ). A presentative result is listed in Table 7, which clearly shows $O\left(\tau^{2}\right)$ rate. This result is consistent with the theoretical analysis, since in this case $O\left(\tau^{2}+h^{2}\right)=O\left(\tau^{2}\right)$ when we fix $\tau=0.01 h$.

\subsection{Example 3: Wave propagation in a rectangular metamaterial slab}

Here we consider a wave propagation model originally introduced and solved by FDTD method by Ziolkowski [21]. In this example, a metamaterial slab is chosen to be [0.024, 0.054] $m \times[0.002,0.062] \mathrm{m}$, which is located inside a vacuum with dimension $[0,0.07] m \times[0,0.064] \mathrm{m}$. The vacuum is surrounded by a PML with thickness $d d=12 h$, where $h$ denotes the mesh size. The 2 -D transverse magnetic

Table 5: Example 2. $L_{\infty}$ and $L_{2}$ errors for magnetic field $H_{x}$ with cubic basis function and $\tau=10^{-12}$ at one time step.

\begin{tabular}{|c|c|c|c|c|c|c|c|c|c|}
\hline & $h=1 / 5$ & $h=1 / 10$ & Rates & $h=1 / 20$ & Rates & $h=1 / 40$ & Rates & $h=1 / 80$ & Rates \\
\hline$L_{\infty}$ errors & $9.5958 \mathrm{e}-12$ & $1.4932 \mathrm{e}-12$ & 2.6840 & $2.0184 \mathrm{e}-13$ & 2.8871 & $2.5757 \mathrm{e}-14$ & 2.9702 & $3.2474 \mathrm{e}-15$ & 2.9876 \\
$L_{2}$ errors & $1.2339 \mathrm{e}-12$ & $1.8039 \mathrm{e}-13$ & 2.7740 & $2.3722 \mathrm{e}-14$ & 2.9268 & $2.9871 \mathrm{e}-15$ & 2.9894 & $3.7331 \mathrm{e}-16$ & 3.0003 \\
\hline
\end{tabular}


Table 6: Example 2. $L_{\infty}$ and $L_{2}$ errors for magnetic field $H_{x}$ with cubic basis function and $\tau=10^{-12}$ at 1000 time step.

\begin{tabular}{|c|c|c|c|c|c|c|c|c|c|}
\hline & $h=1 / 5$ & $h=1 / 10$ & Rates & $h=1 / 20$ & Rates & $h=1 / 40$ & Rates & $h=1 / 80$ & Rates \\
\hline$L_{\infty}$ errors & $9.5958 \mathrm{e}-9$ & $1.4933 \mathrm{e}-9$ & 2.6839 & $2.0183 \mathrm{e}-10$ & 2.8873 & $2.5762 \mathrm{e}-11$ & 2.9698 & $3.2503 \mathrm{e}-12$ & 2.9866 \\
$L_{2}$ errors & $1.2339 \mathrm{e}-9$ & $1.8039 \mathrm{e}-10$ & 2.7740 & $2.3721 \mathrm{e}-11$ & 2.9269 & $2.9867 \mathrm{e}-12$ & 2.9895 & $3.7260 \mathrm{e}-13$ & 3.0029 \\
\hline
\end{tabular}

Table 7: Example 2. $L_{\infty}$ and $L_{2}$ errors for magnetic field $H_{x}$ with qudratic basis function, $\tau=0.01 h$ and $n t=10 / h$ time steps.

\begin{tabular}{|c|c|c|c|c|c|c|c|c|c|}
\hline & $h=1 / 5$ & $h=1 / 10$ & Rates & $h=1 / 20$ & Rates & $h=1 / 40$ & Rates & $h=1 / 80$ & Rates \\
\hline$L_{\infty}$ errors & 0.6144 & 0.1807 & 1.7656 & 0.0394 & 2.1973 & 0.0112 & 1.8147 & 0.0028 & 2.0 \\
$L_{2}$ errors & 0.0810 & 0.0216 & 1.9069 & 0.0042 & 2.3626 & $9.7398 \mathrm{e}-004$ & 2.1084 & $2.4353 \mathrm{e}-004$ & 1.9998 \\
\hline
\end{tabular}

PML model can be obtained from (51)-(54):

$$
\begin{aligned}
& \mu_{0} \frac{\partial H_{x}}{\partial t}=-\frac{\partial E_{z}}{\partial y}-K_{x}+\mu_{0}\left(\sigma_{x}-\sigma_{y}\right) H_{x} \\
& \mu_{0} \frac{\partial H_{y}}{\partial t}=\frac{\partial E_{z}}{\partial x}-K_{y}-\mu_{0}\left(\sigma_{x}-\sigma_{y}\right) H_{y} \\
& \epsilon_{0} \frac{\partial E_{z}}{\partial t}=\frac{\partial H_{y}}{\partial x}-\frac{\partial H_{x}}{\partial y}-J_{z}-\epsilon_{0}\left(\sigma_{x}+\sigma_{y}\right) E_{z} \\
& \frac{\partial J_{z}}{\partial t}=\epsilon_{0} \sigma_{x} \sigma_{y} E_{z} \\
& \frac{\partial K_{x}}{\partial t}=-\sigma_{x} K_{x}+\mu_{0}\left(\sigma_{x}-\sigma_{y}\right) \sigma_{x} H_{x} \\
& \frac{\partial K_{y}}{\partial t}=-\sigma_{y} K_{y}-\mu_{0}\left(\sigma_{x}-\sigma_{y}\right) \sigma_{y} H_{y}
\end{aligned}
$$

where the subscripts ' $\mathrm{x}, \mathrm{y}$ ' and ' $\mathrm{z}$ ' denote the corresponding components.

The incident source wave is imposed as $E_{z}$ field and is excited at $x=0.004 m$ and $y \in$ $[0.025,0.035] m$. The source wave varies in space as $e^{-(x-0.03)^{2} /(50 h)^{2}}$ and in time as [21]:

$$
f(t)= \begin{cases}0, & \text { for } t<0 \\ g_{1}(t) \sin \left(\omega_{0} t\right), & \text { for } 0<t<m T_{p} \\ \sin \left(\omega_{0} t\right), & \text { for } m T_{p}<t<(m+k) T_{p} \\ g_{2}(t) \sin \left(\omega_{0} t\right), & \text { for }(m+k) T_{p}<t<(2 m+k) T_{p} \\ 0, & \text { for } t>(2 m+k) T_{p}\end{cases}
$$

where we denote $T_{p}=1 / f_{0}$, and

$$
\begin{gathered}
g_{1}(t)=10 x_{1}^{3}-15 x_{1}^{4}+6 x_{1}^{5}, \quad x_{1}=t / m T_{p}, \\
g_{2}(t)=1-\left(10 x_{2}^{3}-15 x_{2}^{4}+6 x_{2}^{5}\right), \quad x_{2}=\left(t-(m+k) T_{p}\right) / m T_{p} .
\end{gathered}
$$


In our simulation, we use $m=2, k=100$.

The damping function $\sigma_{x}$ and $\sigma_{x}$ are chosen as a fouth order polynomial function, more specifically, we choose:

$$
\sigma_{x}(x, y)=\left\{\begin{array}{cc}
\sigma_{\max }\left(\frac{x-0.07}{d d}\right)^{4} & \text { if } x \geq 0.07 \\
\sigma_{\max }\left(\frac{|x|}{d d}\right)^{4} & \text { if } x \leq 0.0 \\
0 & \text { elsewhere }
\end{array}\right.
$$

where $\sigma_{\max }=-\log (\mathrm{err}) * 5 * 0.07 * c_{v} /(2 * d d)$ with $\mathrm{err}=10^{-7}$. Function $\sigma_{y}$ has the same form but varies with respect to the $y$ variable.

An unstructured triangular mesh with 14586 triangles and 7422 nodes is used for our simulation. The mesh size $h=2 * 10^{-4}$ and the time step size $\tau=0.1 \mathrm{ps}$ are used. The mesh and the absolute value of $E_{z}$ field at various times are presented in Fig. 1, which clearly shows that the wave propagates backwards in the metamaterial slab and demonstrates the re-focusing property of metamaterials. The simulation is consistent with results obtained by the FDTD method [21] and the finite element method with edge elements [10]. Moreover, the leap-frog DG method is quite efficient as evidenced by our implementation that the CPU times are 172.71s, 342.80, 510.50s. 681.63s and 854.14s for 1000, 2000, 3000, 4000, and 5000 time step simulations, respectively.

\subsection{Example 4: Wave propagation in a triangular metamaterial slab}

The setup of this model is basically same as the last example, the only difference is that the rectangular metamaterial slab is replaced by a triangular slab with vertices $(0.024,0.002),(0.054,0.002)$, and $(0.024,0.062)$. A similar example was developed [10] to demonstrate Snell's Law and the negative refractive index of the metamaterial. An unstructured triangular mesh with 14600 triangles and 7429 nodes is used for this model. The mesh size $h=2 * 10^{-4}$ and the time step size $\tau=0.1 p s$ are used. The mesh and $E_{z}$ fields at various times are presented in Fig. 2, which clearly shows that the wave bends toward the same side at the interface between the metamaterial and the vacuum by obeying Snell's Law. The simulation is consistent with results obtained by edge elements [10]. Furthermore, the leap-frog DG method seems more powerful and efficient compared to our edge element algorithm with hybrid grids. The CPU times of our DG method are 173.20s, 347.58, 519.39s. 691.69s and 864.14s for 1000, 2000, 3000, 4000, and 5000 time step simulations, respectively.

\section{Conclusions}

In this paper, we develop a nodal discontinuous Galerkin method for solving the time-dependent Maxwells equations in metamaterials. We prove the numerical stability and error estimate for both semi- and fully-discrete schemes. Numerical results with analytical solutions are first presented to support the theoretical analysis and check the correctness of our algorithmic implementation. Then two wave propagation problems are presented to illustrate the interesting backward propagation phenomenon happened when wave propagates in metamaterials.

\section{References}

[1] B. Cockburn, G.E. Karniadakis and C.-W. Shu (eds.), Discontinuous Galerkin Methods: Theory, Computation and Applications, Springer, Berlin, 2000. 

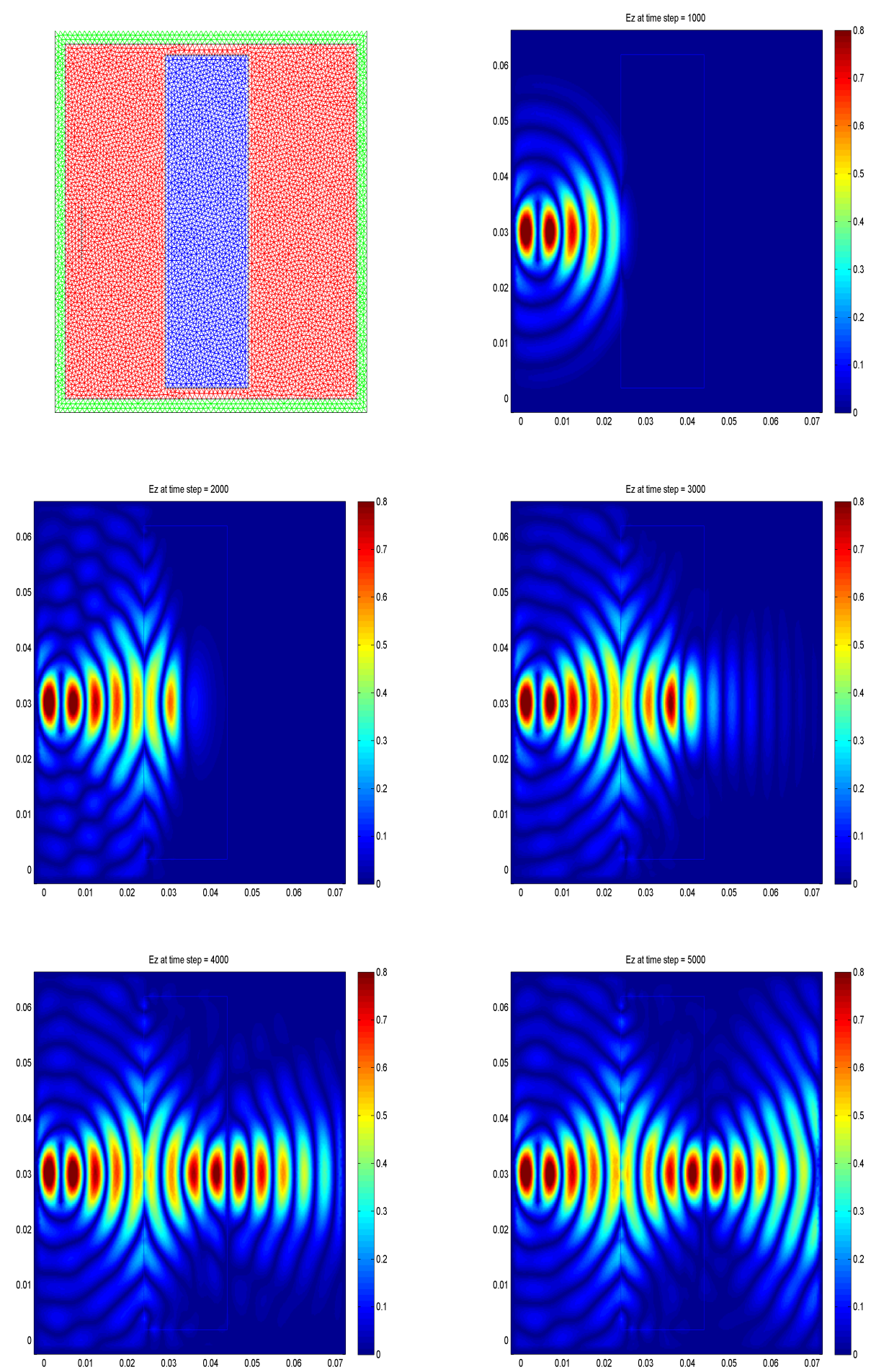

Figure 1: Example 3. The mesh and contour plots of $\left|E_{z}\right|$ obtained with $h=2 \cdot 10^{-4}, \tau=0.1 p s$ at 1000, 2000, 3000, 4000, and 5000 time steps. 

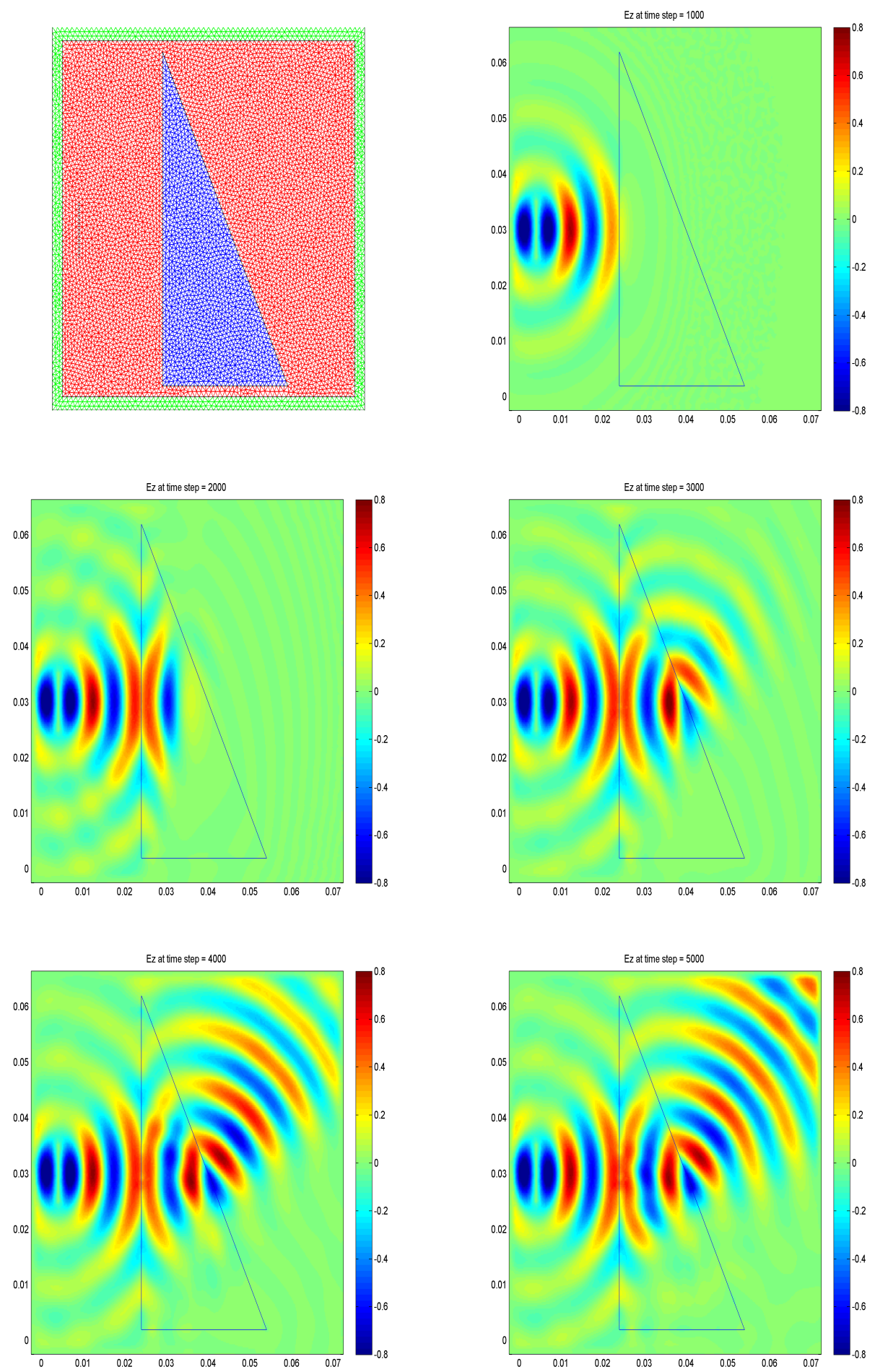

Figure 2: Example 4. The mesh and contour plots of $E_{z}$ obtained with $h=2 \cdot 10^{-4}, \tau=0.1 p s$ at 1000, 2000, 3000, 4000, and 5000 time steps. 
[2] B. Cockburn, F. Li and C.-W. Shu, Locally divergence-free discontinuous Galerkin methods for the Maxwell equations, J. Comp. Phys. 194 (2004) 588-610.

[3] T.J. Cui, D.R. Smith and R. Liu (eds.), Metamaterials: Theory, Design, and Applications, Springer, New York, 2009.

[4] N. Engheta and R.W. Ziolkowski (eds.), Electromagnetic Metamaterials: Physics and Engineering Explorations, Wiley-IEEE Press, Hoboken, NJ, 2006.

[5] L. Fezoui, S. Lanteri, S. Lohrengel and S. Piperno, Convergence and stability of a discontinuous Galerkin time-domain methods for the 3D heterogeneous Maxwell equations on unstructured meshes, Model. Math. Anal. Numer. 39 (6) (2005) 1149-1176.

[6] M.J. Grote, A. Schneebeli and D. Schötzau, Interior penalty discontinuous Galerkin method for Maxwell's equations: energy norm error estimates, J. Comput. Appl. Math. 204 (2007) 375-386.

[7] J.S. Hesthaven and T. Warburton, Nodal high-order methods on unstructured grids. I. Timedomain solution of Maxwell's equations, J. Comput. Phys. 181 (2002) 186-221.

[8] J.S. Hesthaven and T. Warburton, Nodal Discontinuous Galerkin Methods: Algorithms, Analysis, and Applications, Springer, New York, 2008.

[9] P. Houston, I. Perugia, A. Schneebeli and D. Schötzau, Interior penalty method for the indefinite time-harmonic Maxwell equations, Numer. Math. 100 (2005) 485-518.

[10] Y. Huang, J. Li and W. Yang, Modeling backward wave propagation in metamaterials by the finite element time domain method, SIAM J. Sci. Comput. (accepted, to appear in 2013).

[11] J. Li, Development of discontinuous Galerkin methods for Maxwell's equations in metamaterials and perfectly matched layers, J. Comp. Appl. Math. 236 (2011) 950-961.

[12] J. Li, Numerical convergence and physical fidelity analysis for Maxwell's equations in metamaterials, Comput. Methods Appl. Mech. Engrg. 198 (2009) 3161-3172.

[13] J. Li and Y. Huang, Time-Domain Finite Element Methods for Maxwell's Equations in Metamaterials, Springer Series in Computational Mathematics, vol.43, Springer, 2013.

[14] T. Lu, P. Zhang and W. Cai, Discontinuous Galerkin methods for dispersive and lossy Maxwell's equations and PML boundary conditions, J. Comp. Phys. 200 (2004) 549-580.

[15] E. Montseny, S. Pernet, X. Ferriéres and G. Cohen, Dissipative terms and local time-stepping improvements in a spatial high order Discontinuous Galerkin scheme for the time-domain Maxwell's equations, J. Comp. Phys. 227 (2008) 6795-6820.

[16] C. Scheid and S. Lanteri, Convergence of a discontinuous Galerkin scheme for the mixed time domain Maxwell's equations in dispersive media, IMA J. Numer. Anal. (2012) doi: 10.1093/imanum/drs008. 
[17] D.R. Smith, W.J. Padilla, D.C. Vier, S.C. Nemat-Nasser and S. Schultz, Composite medium with simultaneously negative permeability and permittivity, Phys. Rev. Lett. 84 (2000) 41844187 .

[18] E. Turkel and A. Yefet, Absorbing PML boundary layers for wave-like equations, Appl. Numer. Math. 27 (1998) 533-557.

[19] B. Wang, Z. Xie and Z. Zhang, Error analysis of a discontinuous Galerkin method for Maxwell equations in dispersive media, J. Comp. Phys. 229 (2010) 8552-8563.

[20] R.W. Ziolkowski, Maxwellian material based absorbing boundary conditions, Comput. Methods Appl. Mech. Engrg. 169 (1999), 237-262.

[21] R.W. Ziolkowski, Pulsed and CW Gaussian beam interactions with double negative metamaterial slabs, Optical Express 11 (2003) 662-681. 